\title{
MULTI-INVARIANT SETS \\ ON COMPACT ABELIAN GROUPS
}

BY

DANIEL BEREND

\begin{abstract}
Let $G$ be a finite-dimensional connected compact abelian group. Generalizing previous results, dealing with the case of finite-dimensional tori, a full characterization is given herewith of those commutative semigroups $\Sigma$ of continuous endomorphisms of $G$ which satisfy the following property: The only infinite closed $\Sigma$-invariant subset of $G$ is $G$ itself.
\end{abstract}

Introduction. A multiplicative semigroup of integers $\Sigma$ is lacunary if all its positive elements are powers of a single integer $a$, and nonlacunary otherwise. In [5, p. 48] Furstenberg proves:

THEOREM. If $\Sigma$ is a nonlacunary semigroup of integers and $\alpha$ is an irrational, then $\Sigma \alpha$ is dense modulo 1 .

The theorem gives a partial answer to the following question in the theory of diophantine approximations: Which sets $S$ of positive integers have the property that any irrational number $\alpha$ can be approximated by a rational number $m / s$ with $s \in S$ so that $|\alpha-m / s|<\varepsilon / s$, where $\varepsilon>0$ is arbitrarily small? The case where $S$ is a multiplicative semigroup is completely settled by the theorem. We note that the theorem forms a generalization of a result of Hardy and Littlewood by which if $r$ is a fixed positive integer and $\alpha$ is an irrational, then $\left\{n^{r} \alpha \mid n \in \mathbf{N}\right\}$ is dense modulo 1. Further connections with the theory of diophantine approximations are discussed in [6].

From the point of view of the theory of dynamical systems we have a semigroup of continuous endomorphisms of the circle group $\mathbf{T}$. The theorem implies that, unless $\Sigma$ is a "one-parameter" semigroup, the orbit of every nontorsion element is dense in the group.

The theorem is almost equivalent to the assertion that nonlacunarity is a necessary and sufficient condition for a semigroup $\Sigma$ to have the property that the only infinite closed $\Sigma$-invariant subset of $T$ is $T$ itself. In [1] the conditions for a commutative semigroup $\Sigma$ of endomorphisms of $\mathbf{T}^{r}$ to possess this property are investigated. The following result is obtained there.

Received by the editors March 24, 1983.

1980 Mathematics Subject Classification. Primary 22D40, 54H20; Secondary 10F10, 10F45, 28 A65.

Kev words and phrases. Flow, multi-parameter flow, Thflow, minimal flow, invariant set, solenoid, finite-dimensional group, semigroup of endomorphisms, ergodic endomorphism.

(C)1984 American Mathematical Society $0002-9947 / 84 \$ 1.00+\$ .25$ per page 
THEOREM. $\Sigma$ has the aforementioned property iff it satisfies the following conditions:

(1) There exists some $\sigma \in \Sigma$ such that the characteristic polynomial of $\sigma^{n}$ is irreducible over $\mathbf{Z}$ for every positive integer $n$.

(2) For every common eigenvector $v$ of $\Sigma$ there exists some $\sigma_{\sigma^{*}} \in \Sigma$ such that the corresponding eigenvalue $\lambda_{1}$, of $\sigma_{l}$ lies outside the unit disc in the complex plane.

(3) There exist two nonzero endomorphisms $\sigma$ and $\tau$ in $\Sigma$ such that $\sigma^{\prime}=\tau^{m}$ with $l, m \in \mathbf{Z}$ implies $l=m=0$.

In this paper we extend the class of groups that are examined. Given any finite-dimensional connected compact abelian group, a full characterization is given of those commutative semigroups of endomorphisms having the property in question.

In Chapter I a general framework is outlined for the study of semigroups of continuous transformations of compact metric spaces. Some results of Furstenberg [5], relating originally to one-parameter semigroups, are proved in this more general setup.

The class of groups under examination can be characterized as that of duals of torsion-free discrete abelian groups of finite rank. We divide their study into four steps. First, in Chapter II, the $a$-adic multi-solenoid, that is the dual group of $\mathbf{Z}[1 / a]^{r}$, is dealt with. Several important facts concerning this group are presented, after which the main theorem is formulated (Theorem II.1). The main theme of the proof is carrying out the $p$-adic analogues of considerations held in the course of proving the case of $\mathbf{T}^{r}$ employing archimedean valuations.

In Chapter III we settle the case of groups which are obtained as quotient groups of those considered in Chapter II. It comes out that, unlike the case of $a$-adic multi-solenoids, where semigroups of endomorphisms with the sought property always exist, most of the groups studied in this chapter admit no such semigroups.

Chapter IV is concerned with groups whose duals are of the form $\Lambda^{r}, \Lambda$ being a rank 1 torsion-free group of idempotent type. In Chapter $V$ we arrive at the most general case. The groups considered here are quotient groups of those dealt with in the former chapter. The work in both these chapters relies heavily upon the fact that the groups in question can be represented as inverse limits of groups studied in former chapters.

I wish to express my gratitude to $\mathrm{H}$. Furstenberg for his helpful advice concerning the problems discussed here.

\section{CHAPTER I. MULTI - PARAMETER FLOWS}

1. Disjointness. A flow is a couple $(\Omega, \Sigma)$, where $\Omega$ is a compact metric space and $\Sigma$ a semigroup of continuous transformations of $\Omega$ into itself. Assume for simplicity that $\Sigma$ contains a unit element $I$ acting on $\Omega$ as the identity. If $\Sigma$ is generated by a single transformation $T$, then the flow is denoted by $(\Omega, T)$. In this chapter we present a few results, proved in [5] in the case of "one-parameter" flows $(\Omega, T)$, for "multi-parameter" flows $(\Omega, \Sigma)$.

Let $(\Omega, \Sigma)$ be a flow. A set $A \subseteq \Omega$ is $\Sigma$-invariant if $\Sigma A \subseteq A$, where $\Sigma A=$ $\bigcup_{\sigma \in \Sigma} \sigma(A)$. A closed $\Sigma$-invariant set $A$ defines a subflow $(A, \Sigma)$, where $\Sigma$ now 
denotes the original semigroup of transformations restricted to $A$. Given a point $\xi \in \Omega$, the set $\Sigma \xi$ is the orbit of $\xi$.

Let $X=\left(\Omega_{X}, \Sigma\right)$ and $Y=\left(\Omega_{Y}, \Sigma\right)$ be two flows with the same acting semigroup $\Sigma$. A homomorphism of $X$ onto $Y$ is a continuous mapping $\varphi$ of $\Omega_{X}$ onto $\Omega_{Y}$ commuting with $\varphi$, i.e. such that $\sigma(\varphi \xi)=\varphi(\sigma \xi)$ for $\sigma \in \Sigma, \xi \in \Omega .$. We denote $X \stackrel{\varphi}{\rightarrow} Y$ and say that $Y$ is a factor of $X$. The product of the flows $X$ and $Y$ is denoted by $X \times Y$ and defined as the couple $\left(\Omega_{X} \times \Omega_{Y}, \Sigma\right)$, the action of $\Sigma$ on $\Omega_{X} \times \Omega_{Y}$ being given by

$$
\sigma(\xi, \eta)=(\sigma \xi, \sigma \eta), \quad \sigma \in \Sigma, \xi \in \Omega_{X}, \eta \in \Omega_{Y} .
$$

$X$ and $Y$ are factors of $X \times Y$ under the projections $\pi_{X}$ and $\pi_{Y}$, respectively.

Definition 1.1. The flows $X=\left(\Omega_{X}, \Sigma\right)$ and $Y=\left(\Omega_{Y}, \Sigma\right)$ are disjoint if given any flow $Z=\left(\Omega_{Z}, \Sigma\right)$ and homomorphisms $Z \stackrel{\alpha}{\rightarrow} X$ and $Z \stackrel{\beta}{\rightarrow} Y$, there exists a homomorphism $Z \stackrel{\gamma}{\rightarrow} X \times Y$ with $\alpha=\pi_{X} \gamma$ and $\beta=\pi_{Y} \gamma$.

LEMMA I.1. The flows $X$ and $Y$ are disjoint iff the only closed subset $\Delta \subseteq \Omega_{X} \times \Omega_{Y}$ satisfying (1) $\Sigma \Delta \subseteq \Delta$, (2) $\pi_{X} \Delta=\Omega_{X}$, (3) $\pi_{Y} \Delta=\Omega_{Y}$, is $\Delta=\Omega_{X} \times \Omega_{Y}$.

The proof bears no difference relative to the one-parameter case [5, Lemma II.1].

Lemma 1.2. Let $X$ and $Y$ be disjoint flows, $Z$ any flow. Suppose there is a homomorphism $X \times Z \stackrel{\gamma}{\rightarrow} Y$. Then for every $\xi \in \Omega_{X}$ the mapping $\zeta \rightarrow \gamma(\xi, \zeta)$ carries $\Omega_{Z}$ onto $\Omega_{Y}$.

Again, the proof is exactly as in the one-parameter case [5, Lemma II.2].

Definition 1.2. A flow $(\Omega, \Sigma)$ is ergodic if every closed $\Sigma$-invariant proper subset of $\Omega$ is nowhere dense.

Definition 1.3. A flow $(\Omega, \Sigma)$ is minimal if it has no proper subflow. A closed $\Sigma$-invariant set $A \subseteq \Omega$ is minimal if the subflow $(A, \Sigma)$ is minimal.

DEFINITION I.4. $X=(\Omega, \Sigma)$ is an $\mathscr{F}_{\text {fflow }}$ if there exists a sequence of subsemigroups of $\Sigma, \Sigma=\Sigma^{(1)} \supseteq \Sigma^{(2)} \supseteq \cdots$, with $I \in \Sigma^{(k)}$ for each $k$, such that:

(1) The flow $\left(\Omega, \Sigma^{(k)}\right)$ is ergodic for every $k \geqslant 1$.

(2) $\Sigma / \Sigma^{(k)}$ has a finite group structure in the following sense: $\Sigma$ decomposes into a disjoint union

$$
\Sigma=\Sigma_{1}^{(k)} \cup \Sigma_{2}^{(k)} \cup \cdots \cup \Sigma_{n_{k}}^{(k)} \quad\left(\Sigma_{1}^{(k)}=\Sigma^{(k)}\right),
$$

such that for every $1 \leqslant i, j \leqslant n_{k}$ there exists some $1 \leqslant h \leqslant n_{k}$ with $\Sigma_{i}^{(k)} \Sigma_{j}^{(k)} \subset \Sigma_{h}^{(k)}$. The collection $\left\{\Sigma_{i}^{(k)} \mid 1 \leqslant i \leqslant n_{k}\right\}$ with the operation $\circ$ defined by $\Sigma_{i}^{(k)} \circ \Sigma_{j}^{(k)}=\Sigma_{h}^{(k)}$ for that $h$ forms a finite group ( $\Sigma^{(k)}$ being its unit).

(3) The collection of fixed points of $\Sigma^{(k)}$ becomes dense:

$$
\overline{\bigcup_{k=1}^{x}\left\{\xi \in \Omega \mid \Sigma^{(k)} \xi=\{\xi\}\right\}}=\Omega .
$$

We note that if $(\Omega, \Sigma)$ is an $\mathscr{F}$ flow then every $\sigma \in \Sigma$ is surjective. In fact, since $\sigma^{n_{k}} \in \Sigma^{(k)}$, every fixed point of $\Sigma^{(k)}$ belongs to the image of $\sigma$. Since the collection of all fixed points is dense, $\sigma$ is surjective. 
THEOREM I.1. Let $X=\left(\Omega_{X}, \Sigma\right)$ be an Thflow and $Y=\left(\Omega_{Y}, \Sigma\right)$ a minimal flow. Then $X$ and $Y$ are disjoint.

PROOF. We employ the disjointness criterion given by Lemma I.1. Let $\Delta \subseteq \Omega_{X} \times \Omega_{Y}$ be a closed $\Sigma$-invariant set, the projections of which on $\Omega_{X}$ and $\Omega_{Y}$ are $\Omega_{X}$ and $\Omega_{Y}$, respectively. It has to be shown that $\Delta=\Omega_{X} \times \Omega_{Y}$.

Let $\left(\Sigma^{(k)}\right)_{k=1}^{\infty}$ be a sequence of subsemigroups of $\Sigma$ satisfying the conditions of Definition I.4 for the $\mathscr{R}_{\text {flow }} X$. Choose inductively a sequence of subsets of $\Omega_{Y}$,

$$
\Omega_{Y}=\Omega_{Y}^{(1)} \supseteq \Omega_{Y}^{(2)} \supseteq \cdots,
$$

such that $\Omega_{Y}^{(k)}$ is $\Sigma^{(k)}$-minimal. The set $\Omega_{Y}^{(\infty)}=\bigcap_{k=1}^{\infty} \Omega_{Y}^{(k)}$ is closed and nonvoid.

For fixed $k$ define the sets

$$
\Lambda_{k, i}=\left\{\xi \in \Omega_{X} \boxminus \eta \in \overline{\Sigma_{i}^{(k)} \Omega_{Y}^{(k)}},(\xi, \eta) \in \Delta\right\}, \quad 1 \leqslant i \leqslant n,
$$

where $n=n_{k}$ is as in Definition I.4. It is evident that $\bigcup_{i=1}^{n} \overline{\sum_{i}^{(k)} \Omega_{Y}^{(k)}}$ is a closed $\sum$-invariant subset of $\Omega_{Y}$, and the minimality of $Y$ implies $\bigcup_{i=1}^{n} \overline{\Sigma_{i}^{(k)} \Omega_{Y}^{(k)}}=\Omega_{Y}$. We therefore have $\bigcup_{i=1}^{n} \Lambda_{k, i}=\Omega_{X}$. Since the sets $\Lambda_{k, i}$ are closed one of them, say $\Lambda_{k, j}$, has a nonempty interior. Obviously $\Lambda_{k, i}$ is $\Sigma^{(k)}$-invariant for each $i$, and by the ergodicity of $\left(\Omega_{X}, \Sigma^{(k)}\right)$ we obtain $\Lambda_{k, j}=\Omega_{X}$.

Set

$$
F_{k}=\left\{\xi \in \Omega_{X} \mid \Sigma^{(k)} \xi=\{\xi\}\right\}
$$

We easily see that

$$
\Lambda_{k, 1}=\Sigma_{j}^{(k)^{n}} \Lambda_{k, 1} \supseteq \Sigma_{j}^{(k)^{n-1}} \Lambda_{k, j} \supseteq \Sigma_{j}^{(k)^{n-1}}\left(\Sigma_{j}^{(k)} F_{k}\right)=F_{k} .
$$

Thus the sets in the descending sequence $\left(\Lambda_{k, 1}\right)_{k=1}^{\infty}$ contain the corresponding sets in the ascending sequence $\left(F_{k}\right)_{k=1}^{\infty}$. Since $\overline{\bigcup_{k=1}^{\infty} F_{k}}=\Omega_{X}$, it follows that $\Lambda_{k, 1}=\Omega_{X}$ for every $k$. That is, for every $\xi \in \Omega_{X}$ and $k \geqslant 1$ we have $(\xi, \eta) \in \Delta$ for some $\eta \in \Omega_{Y}^{(k)}$. We conclude that for every $\xi \in \Omega_{X}$ there exists some $\eta \in \Omega_{Y}^{(\infty)}$ with $(\xi, \eta) \in \Delta$.

Now fix $k$. Let $\xi$ be an element of $F_{k}$. Select $\eta \in \Omega_{Y}^{(\infty)}$ with $(\xi, \eta) \in \Delta$. Since $\Delta$ is $\sum$-invariant we have

$$
\Delta \supseteq \overline{\Sigma^{(k)}(\xi, \eta)}=\{\xi\} \times \overline{\Sigma^{(k)} \eta}=\{\xi\} \times \Omega_{Y}^{(k)} \supseteq\{\xi\} \times \Omega_{Y}^{(\infty)} .
$$

Hence

$$
\Delta \supseteq \overline{\bigcup_{k=1}^{\infty} F_{k} \times \Omega_{Y}^{(\infty)}}=\Omega_{X} \times \Omega_{Y}^{(\infty)}
$$

Set

$$
\Omega_{Y}^{\prime}=\left\{\eta \in \Omega_{Y} \mid \Omega_{X} \times\{\eta\} \subseteq \Delta\right\} .
$$

The set $\Omega_{Y}^{\prime}$ contains $\Omega_{Y}^{(\infty)}$, whence it is nonvoid. Since the transformations in $\Sigma$ are surjective as transformations of $\Omega_{X}, \Omega_{Y}^{\prime}$ is $\Sigma$-invariant. $\Omega_{Y}^{\prime}$ is also evidently closed. The minimality of $Y$ implies therefore $\Omega_{Y}^{\prime}=\Omega_{Y}$. Thus $\Delta=\Omega_{X} \times \Omega_{Y}$, which completes the proof.

2. Group flows. A flow $(G, \Sigma)$, where $G$ is a compact metric abelian group and $\Sigma$ a semigroup of continuous endomorphisms, is a group flow. 
Definition 1.5. Let $(G, \Sigma)$ be a group flow. A closed $\Sigma$-invariant set $A \subseteq G$ is restricted if the only closed $\Sigma$-invariant set $B \subseteq G$ for which $A+B=G$ is $B=G$.

THEOREM I.2. If $(G, \Sigma)$ is a group $\mathscr{R}$ flow, then every minimal set is restricted.

Proof. Let $M$ be a minimal set and $B$ a closed $\Sigma$-invariant set with $M+B=G$. We shall employ Lemma $I .2$ for the flows $X=(M, \Sigma), Y=(G, \Sigma)$ and $Z=(B, \Sigma)$. Consider the homomorphism $X \times Z \stackrel{\gamma}{\rightarrow} Y$ defined by

$$
\gamma(\mu, \beta)=\mu+\beta, \quad \mu \in M, \beta \in B .
$$

Since $X$ is minimal and $Y$ an $\mathscr{r}$ flow, it follows from Theorem $I .1$ that $X$ and $Y$ are disjoint. By Lemma $I .2$ we obtain $\gamma(\mu, B)=G$ for every $\mu \in M$. Hence $B=G$, completing the proof.

A (one-parameter) process is a quadruple $(\Omega, \mathscr{B}, \mu, T)$, where $(\Omega, \mathscr{B}, \mu)$ is a probability space and $T$ a measure-preserving transformation of the space. The process is ergodic if $A \in \mathscr{B}$ and $A T$-invariant implies $\mu(A)=0$ or $\mu(A)=1$. Assume now that $(\Omega, T)$ is a flow, $\mathscr{B}$ is the Borel field of $\Omega$ and the support of $\mu$ is the entire space $\Omega$. In this case the ergodicity of the process $(\Omega, \mathscr{B}, \mu, T)$ implies that of the flow $(\Omega, T)$. In fact, if $A$ is a $T$-invariant proper subset of $\Omega$ then necessarily $\mu(A)=0$, whence $A$ is nowhere dense.

Let $G$ be a compact metric abelian group, $\mathscr{B}$ its Borel field and $\mu$ the Haar measure. $\Gamma=\hat{G}$ is the dual group. It is easy to see that any epimorphism $\sigma$ of $G$ preserves $\mu$. The following theorem (see, for example, [3, p. 76]) characterizes ergodic epimorphisms.

THEOREM I.3. $(G, \mathscr{B}, \mu, \sigma)$ is ergodic iff every nontrivial $\gamma \in \Gamma$ has an infinite orbit in the dual group under the dual endomorphism of $\sigma$.

CHAPTER II. INVARIANT SETS ON MULTI - SOLENOIDS OF FINITE TYPE

1. Generalities and the main theorem. Given a positive integer $a, Z[1 / a]$ denotes the ring obtained from $\mathbf{Z}$ by adjoining $1 / a$. The same notation relates also to the additive group of this ring. Endowed with the discrete topology, $Z[1 / a]$ is a topological group. We may assume $a$ to be square-free, say $a=p_{1} p_{2} \cdots p_{h}$. The dual group is denoted by $\Omega_{a}$ and is called the $a$-adic solenoid. The construction of the $a$-adic solenoid, as well as that of more general solenoids, is described in detail in $[7, \S 10]$. We now survey a few facts concerning $\Omega_{a}$, emphasizing those which are of importance for the study of endormorphisms of $\Omega_{a}$ and $\Omega_{a}^{r}$.

Let $\mathbf{Q}_{a}$ and $\mathbf{Z}_{a}$ denote the ring of $a$-adic numbers and its subring of $a$-adic integers, respectively. (The definitions and basic properties of these rings appear, for example, in [9].) Consider the monomorphism

$$
i: \mathbf{Z}[1 / a] \rightarrow \mathbf{R} \times \mathbf{Q}_{a}
$$

given by

$$
i(s)=(s, s), \quad s \in \mathbf{Z}[1 / a] .
$$

It is easy to verify that $i$ forms a discrete embedding of $\mathbf{Z}[1 / a]$ in $\mathbf{R} \times \mathbf{Q}_{a}$, and hence the former group may be considered as a subgroup of the latter. To study the dual endomorphism we recall that $\hat{\mathbf{R}}$ is topologically isomorphic with $\mathbf{R}$, the action 
of the character $\chi_{x}$ corresponding to $x \in \mathbf{R}$ being given by $\chi_{x}(y)=\exp (2 \pi i x y)$ for $y \in \mathbf{R}$. Similarly, $\hat{\mathbf{Q}}_{a}$ is topologically isomorphic with $\mathbf{Q}_{a}$ and the action is given by $\chi_{x}(y)=\exp (2 \pi i\{x y\})$, where $\{z\}$ denotes the "fractional part" of $z \in \mathbf{Q}_{a}$. The dual endomorphism

$$
\pi=\hat{i}: \mathbf{R} \times \mathbf{Q}_{a} \rightarrow \Omega_{a}
$$

gives therefore $\Omega_{a}$ as a quotient group of $\mathbf{R} \times \mathbf{Q}_{a}$.

We now claim that

$$
\operatorname{Ker}(\pi)=\{(s,-s) \mid s \in \mathbf{Z}[1 / a]\} .
$$

In fact, this follows in a routine manner from $\operatorname{Ker}(\pi)=A\left(\mathbf{R} \times \mathbf{Q}_{a}, \operatorname{Im}(i)\right)$, where, given a closed subgroup $H$ of a locally compact abelian group $G, A(\hat{G}, H)$ denotes the annihilator of $H$ in $\hat{G}$.

From the preceding remarks we easily conclude that $\Omega_{a}$ can be concretely interpreted in the following way. To each $x \in \Omega_{a}$ there corresponds a unique $(y, z) \in[0,1) \times \mathbf{Z}_{a} \subseteq \mathbf{R} \times \mathbf{Q}_{a}$ carried to $x$ by $\pi$. If to $x_{1}, x_{2} \in \Omega_{a}$ there correspond $\left(y_{1}, z_{1}\right),\left(y_{2}, z_{2}\right) \in[0,1) \times \mathbf{Z}_{a}$ then to $x_{1}+x_{2}$ there corresponds $\left(y_{1}+y_{2}-\right.$ $\left.\left[y_{1}+y_{2}\right], z_{1}+z_{2}+\left[y_{1}+y_{2}\right]\right)$. Thus $\Omega_{a}$ consists of the set $[0,1) \times \mathbf{Z}_{a}$ with the described operation, and the collection of all sets

$$
U_{n}=[0,1 / n) \times a^{n} \mathbf{Z}_{a} \cup(1-1 / n, 1) \times\left(-1+a^{n} \mathbf{Z}_{a}\right)
$$

with $n \in \mathbf{N}$ forms a basis for the open neighbourhoods of 0 . In a slightly different manner $\Omega_{a}$ may be thought of as the set of all doubly-infinite sequences with entries in $\{0,1, \ldots, a-1\}$, where the "left half" of any sequence is an $a$-adic integer and the "right half" a real number in $[0,1]$ expanded to the base $a$. (Note that certain pairs of sequences are to be identified, for instance

$$
(\ldots, 0,0,0, a-1, a-1, \ldots) \text { and }(\ldots, 0,0,1,0,0, \ldots) .)
$$

We turn to study the ring $\operatorname{End}\left(\Omega_{a}\right)$ of all continuous endomorphisms of $\Omega_{a}$. The following lemma will be useful for this and similar purposes.

LEMMA II.1. Let $G$ be a locally compact abelian group, $H$ a closed subgroup of $G$, $\Gamma=\hat{G}$ and $\Lambda=A(\Gamma, H)$. A continuous endomorphism $\sigma$ of $G / H$ can be lifted to $G$ iff the dual endomorphism $\hat{\sigma}$ of $\Lambda$ can be extended to $\Gamma$. The lifting is unique iff the extension is.

The lemma is an easy consequence of duality. We shall also use the fact that the liftings to $G$ of two endomorphisms $\sigma, \tau \in \operatorname{End}(G / H)$ commute iff the extensions to $\Gamma$ of $\hat{\boldsymbol{\sigma}}, \hat{\tau} \in \operatorname{End}(\Lambda)$ commute.

It can now be shown that any $\sigma \in \operatorname{End}\left(\Omega_{a}\right)$ has a unique lifting to $\mathbf{R} \times \mathbf{Q}_{a}$. In fact, in view of the lemma it is sufficient to prove that any $\tau \in \operatorname{End}(\operatorname{Im}(i))$ can be uniquely extended to $\mathbf{R} \times \mathbf{Q}_{a}$. First, it is evident that $\tau$ is of the form $\tau_{t}$ for some $t \in \mathbf{Z}[1 / a]$, where

$$
\tau_{t}(s, s)=(s t, s t), \quad s \in \mathbf{Z}[1 / a]
$$

Obviously, $\tau_{t}$ can be extended to $\mathbf{R} \times \mathbf{Q}_{a}$ by

$$
\bar{\tau}_{t}(x, y)=(x t, y t), \quad(x, y) \in \mathbf{R} \times \mathbf{Q}_{a} .
$$


To show the uniqueness of the extension it is sufficient to prove that any $\varphi \in$ $\operatorname{End}\left(\mathbf{R} \times \mathbf{Q}_{a}\right)$ is of the form $\varphi_{u, v}$ for suitable $u \in \mathbf{R}$ and $v \in \mathbf{Q}_{a}$, where

$$
\varphi_{u, c}(x, y)=(x u, y v), \quad(x, y) \in \mathbf{R} \times \mathbf{Q}_{a} .
$$

Denote by $\operatorname{Hom}\left(G_{1}, G_{2}\right)$ the group of all continuous homomorphisms from a locally compact abelian group $G_{1}$ to another one $G_{2}$. We claim that $\operatorname{Hom}\left(\mathbf{R}, \mathbf{Q}_{a}\right)=\{0\}$ and $\operatorname{Hom}\left(\mathbf{Q}_{a}, \mathbf{R}\right)=\{0\}$. The first of these assertions follows from connectedness considerations and the second is deduced from it by passing to dual endomorphisms. (Alternatively, direct proofs can be given.) Since any endomorphism of $\mathbf{R}$ (resp. $\mathbf{Q}_{a}$ ) is of the form $\psi_{w}$ for some $w \in \mathbf{R}$ (resp. $w \in \mathbf{Q}_{a}$ ), where $\psi_{w}(z)=z w$ for all $z \in \mathbf{R}$ (resp. $z \in \mathbf{Q}_{a}$ ), we infer that the endomorphisms of $\mathbf{R} \times \mathbf{Q}_{a}$ are of the indicated form. Thus we obtain the unique lifting possibility of endomorphisms of $\Omega_{a}$ to $\mathbf{R} \times \mathbf{Q}_{a}$.

Our interest in this chapter is focused on commutative semigroups of endomorphisms of $\Omega_{a}^{r}$. This group is a quotient group of $\mathbf{R}^{r} \times \mathbf{Q}_{a}^{r}$. It is easy to verify that

$$
\operatorname{End}\left(\mathbf{R}^{r} \times \mathbf{Q}_{a}^{r}\right) \simeq M_{r}(\mathbf{R}) \times M_{r}\left(\mathbf{Q}_{a}\right),
$$

where $M_{r}(R)$ denotes the ring of $r \times r$ matrices over a ring $R$. As in the onedimensional case one can show that any endomorphism of $\Omega_{a}^{r}$ admits a unique lifting to $\mathbf{R}^{r} \times \mathbf{Q}_{a}^{r}$. We get $\operatorname{End}\left(\Omega_{a}^{r}\right) \simeq M_{r}(\mathbf{Z}[1 / a])$. The action of a character $\gamma=\left(\gamma_{1}, \gamma_{2}, \ldots, \gamma_{r}\right) \in \mathbf{Z}[1 / a]^{r}$ on $\Omega_{a}^{r}$ is given by

$$
\gamma(x)=\exp \left(2 \pi i \sum_{j=1}^{r}\left\{\gamma_{j} x_{j}\right\}\right), \quad x=\left(x_{1}, x_{2}, \ldots, x_{r}\right)^{T} \in \Omega_{a}^{r} .
$$

Commuting endomorphisms of $\boldsymbol{\Omega}_{a}^{r}$ are lifted to commuting endomorphisms of $\mathbf{R}^{r} \times \mathbf{Q}_{a}^{r}$. Elements of $\boldsymbol{\Omega}_{a}^{r}$ will be considered as column vectors, matrices of $\operatorname{End}\left(\Omega_{a}^{r}\right)$ acting upon them from the left. If $\sigma \in \operatorname{End}\left(\Omega_{a}^{r}\right)$, then $\hat{\sigma}$ is given by the same matrix, acting from the right upon elements of $\mathrm{Z}[1 / a]^{r}$, which are considered as row vectors. Hence $\hat{\sigma}$ will also be denoted by $\sigma$.

Recall now that the ring $\mathbf{Q}_{a}$ decomposes into the direct product, taken over the prime divisors $p$ of $a$, of the fields of $p$-adic numbers, i.e. $\mathbf{Q}_{a} \simeq \prod_{j=1}^{h} \mathbf{Q}_{p_{j}}$, and also $\mathbf{Z}_{a} \simeq \prod_{j=1}^{h} \mathbf{Z}_{p}$ (see, for example, [9, Chapter 5]). It can be shown that $\operatorname{Hom}\left(\mathbf{Q}_{p}, \mathbf{Q}_{q}\right)$ $=\{0\}$ for distinct primes $p$ and $q$. Hence $\operatorname{End}\left(\mathbf{Q}_{a}^{r}\right) \simeq \prod_{j=1}^{h} \operatorname{End}\left(\mathbf{Q}_{p}^{r}\right)$. The lifting of an endomorphism $\sigma$ of $\Omega_{a}^{r}$ to $\mathbf{R}^{r} \times \mathbf{Q}_{p_{1}}^{r} \times \cdots \times \mathbf{Q}_{p_{h}}^{r}$ is given by a matrix $\sigma \in$ $M_{r}(\mathbf{Z}[1 / z])$ acting by

$$
\sigma\left(x_{0}, x_{1}, \ldots, x_{h}\right)=\left(\sigma x_{0}, \sigma x_{1}, \ldots, \sigma x_{h}\right) .
$$

To simplify the notations in what follows we put $p_{0}=\infty$, so that $\mathbf{Q}_{p_{0}}=\mathbf{R}$ and $\mathbf{Z}_{p_{0}}=[0,1] \cdot|\cdot|_{j}$ denotes the $p_{j}$-adic valuation on $\mathbf{Q}_{p_{j}}\left(|\cdot|_{0}\right.$ is the usual valuation on R). The same notation will be employed for the unique extension of the valuation to a given finite extension of $\mathbf{Q}_{p}$. The index $j$ will be dropped in case there is only one relevant valuation.

Let $\Sigma$ be a commutative semigroup of endomorphisms of $\Omega_{a}^{r}$.

Definition II.1. $\left(\boldsymbol{\Omega}_{a}^{r}, \boldsymbol{\Sigma}\right)$ is an ID flow if the only infinite closed $\Sigma$-invariant subset of $\Omega_{a}^{r}$ is $\Omega_{a}^{r}$ itself. 
More briefly, $\Sigma$ may be called an ID semigroup.

Definition II.2. Two endomorphisms $\sigma$ and $\tau$ are rationally dependent if $\sigma^{\prime}=\tau^{m}$ for some integers $l$ and $m$, not both of which are 0 . Otherwise, they are rationally independent.

Rational dependence and independence of pairs of nonzero complex numbers, or elements of some other ring, is similarly defined.

The characteristic polynomial and the minimal polynomial of a matrix $\sigma$ are denoted by $f_{\sigma}$ and $m_{\sigma}$, respectively.

The following theorem forms the main result of this chapter. It generalizes the theorem cited in the Introduction.

THEOREM II.1. A commutative semigroup $\Sigma$ of endomorphisms of $\Omega_{a}^{r}$ is ID if and only if the following conditions hold:

(1) There exists an endomorphism $\sigma$ in $\Sigma$ such that $f_{\sigma^{n}}$ is irreducible over $\mathbf{Q}$ for every positive integer $n$.

(2) For every common eigenvector $v$ of $\Sigma$ there exists an endomorphism $\sigma_{l^{\prime}} \in \Sigma$ whose eigenvalue in the direction of $\Sigma$ is of norm greater than 1 .

(3) $\Sigma$ contains a pair of rationally independent endomorphisms.

REMARK II.1. As was pointed out, it is possible to lift $\Sigma$ to a commutative semigroup of endomorphisms of $\prod_{j=0}^{h} \mathbf{Q}_{p}^{r}$, acting as linear transformations on each $\mathbf{Q}_{p}^{r}$. Lemma II.5 will show that the first condition of the theorem guarantees the diagonalizability of $\Sigma$ over a suitable finite extension of $\mathbf{Q}$, hence over suitable finite extensions of the fields $\mathbf{Q}_{p}, 0 \leqslant j \leqslant h$. The vectors of the bases over these extensions with respect to which $\Sigma$ is in diagonal form, $r(h+1)$ vectors in all, are the common eigenvectors of $\Sigma$, mentioned in the second condition of the theorem.

We turn to prove the theorem, demonstrating first the necessity of the conditions. Those steps whose proof is identical to that given for finite-dimensional tori will be omitted.

2. The necessity of the conditions. Throughtout most of this section $\Sigma$ denotes a commutative ID semigroup of endomorphisms of $\Omega_{a}^{r}$. We prove a series of lemmas which together imply that $\Sigma$ satisfies the conditions stated in Theorem II.1.

LEMMA II.2. $m_{\sigma}$ is irreducible over $\mathbf{Q}$ for every $\sigma \in \Sigma$.

Proof. Suppose, to the contrary, that $m_{\sigma}$ is reducible for some $\sigma \in \Sigma$. By [8, Chapter 8] there exists a $\Sigma$-invariant nontrivial proper subspace $\Gamma$ of $\mathbf{Q}^{r}$. Hence $\Gamma \cap \mathbf{Z}[1 / a]^{r}$ is a nontrivial proper $\mathbf{Z}[1 / a]$-submodule of $\mathbf{Z}[1 / a]^{r}$. It follows that $A\left(\Omega_{a}^{r}, \Gamma \cap \mathbf{Z}[1 / a]^{r}\right)$ is an infinite closed $\Sigma$-invariant proper $\mathbf{Z}[1 / a]$-submodule of $\Omega_{a}^{r}$, contradicting the ID property of $\Sigma$. This proves the lemma.

Denote by $\mathbf{A}$ the field of algebraic numbers.

LeMmA II.3. There exists a basis of $\mathbf{A}^{r}$ with respect to which every $\sigma \in \Sigma$ is in diagonal form.

The proof relies only upon the fact that $m_{\sigma}$ is irreducible for every $\sigma \in \Sigma$. It goes exactly as in the toral case [1, Lemma 3.2]. 
Select a basis $v^{(1)}, v^{(2)}, \ldots, v^{(r)}$ satisfying the conditions of the lemma. The eigenvalues of an endomorphism $\sigma \in \Sigma$ corresponding to this basis are denoted by $\lambda_{1, \sigma}, \lambda_{2, \sigma}, \ldots, \lambda_{r, \sigma}$. From Lemma II.2 it follows that $\sigma$ is uniquely determined by $\lambda_{1, \sigma}$. Let $K$ denote the field extension of $\mathbf{Q}$ obtained by adjoining the set $\Lambda_{1}=$ $\left\{\lambda_{1,0} \mid \sigma \in \Sigma\right\}$.

\section{LEMMA II.4. $[K: \mathbf{Q}]=r$.}

Proof. Since any element of $K$ is obviously an eigenvalue of an $r \times r$ rational matrix, and therefore of a degree not exceeding $r$ over $\mathbf{Q}$, we have $[K: \mathbf{Q}] \leqslant r$. Assume that $[K: \mathbf{Q}]=k<r$. Then the (additive) $\mathbf{Z}[1 / a]$-module of endomorphisms $\Sigma^{\prime}$ generated by $\Sigma$ is of rank $k$. Let $\sigma_{1}, \sigma_{2}, \ldots, \sigma_{k}$ be a basis of this module. For an arbitrary rank $1 \mathbf{Z}[1 / a]$-submodule $\Gamma$ of $\mathbf{Z}[1 / a]^{r}$, consider the set $\Gamma^{\prime}=\sum_{i=1}^{k} \Gamma \sigma_{i} . \Gamma^{\prime}$ is a nontrivial $\Sigma$-invariant $\mathbf{Z}[1 / a]$-submodule of $\mathbf{Z}[1 / a]^{r}$, the rank of which is at most $k$. Considering $A\left(\Omega_{a}^{r}, \Gamma^{\prime}\right)$ we obtain therefore a contradiction to $\Sigma$ being ID. This completes the proof.

The necessity of the first condition of the theorem can now be proved.

Proposition II.1. There exists some $\sigma \in \Sigma$ such that $f_{\sigma^{\prime \prime}}$ is irreducible over $\mathbf{Q}$ for every positive integer $n$.

The proof goes along the following lines. Assuming that the proposition fails to be true we arrive at a decomposition of $\Sigma$ of the form

$$
\Sigma=\bigcup_{i=1}^{l} \Sigma_{\sqrt{F_{i}}},
$$

where $F_{1}, F_{2}, \ldots, F_{l}$ are the proper subfields of $K$ and for an arbitrary subset $B$ of $K$ we denote

$$
\sqrt{B}=\left\{\alpha \in K \boxminus n \in \mathbf{N}, \alpha^{n} \in B\right\}, \quad \Sigma_{B}=\left\{\sigma \in \Sigma \mid \lambda_{1, \sigma} \in B\right\} .
$$

Let $\Gamma$ be an arbitrary $\mathbf{Z}[1 / a]$-submodule of $\mathbf{Z}[1 / a]^{r}$ of rank $r-1$. Set $H=A\left(\Omega_{a}^{r}, \Gamma\right)$. The set $\overline{\Sigma H}$ is an infinite closed $\Sigma$-invariant set, and to arrive at a contradiction we have to prove only that it is properly contained in $\Omega_{a}^{r}$. For this it is sufficient to show that for any proper subfield $F$ of $K$ the set $\Sigma_{\sqrt{F}} H$ is contained in the union of finitely many closed proper submodules of $\Omega_{a}^{r}$. We claim that $\sqrt{F}$ can be decomposed as

$$
\sqrt{F}=\bigcup_{j=1}^{m} F \alpha_{j}
$$

for suitable elements $\alpha_{1}, \alpha_{2}, \ldots, \alpha_{m} \in K$. Suppose such a decomposition has been established. Then it remains to prove that $\Sigma_{F \alpha} H$ is contained in a closed proper submodule of $\Omega_{a}^{r}$ for every $\alpha \in K$. It is obvious that the $Z[1 / a]$-module spanned by $\Sigma_{F \alpha}$ is of rank not exceeding $[F: \mathbf{Q}]$. Let $\sigma_{1}, \sigma_{2}, \ldots, \sigma_{s}$ be a basis of this module. The set $\bigcap_{i=1}^{s} \Gamma \sigma_{i}^{-1}$ forms a submodule of $\mathbf{Z}[1 / a]^{r}$ of rank not less than $r-[F: \mathbf{Q}]$, and hence $A\left(\Omega_{a}^{r}, \cap_{i=1}^{s} \Gamma \sigma_{i}^{-1}\right)$ is a proper submodule of $\Omega_{a}^{r}$. Now it is obvious that $\Sigma_{F \alpha} H \subseteq A\left(\Omega_{a}^{r}, \cap_{i=1}^{s} \Gamma \sigma_{i}^{-1}\right)$, which implies that $\overline{\Sigma H}$ is a proper subset of $\Omega_{a}^{r}$.

It is evident that (1) is equivalent to

$$
\left[\sqrt{F^{*}}: F^{*}\right]<\infty
$$


where $F^{*}$ is the multiplicative group of $F$. The proof of (2) depends on classical algebraic number theory and can be found in [1] as the main step in the proof of Proposition 3.1.

Given a polynomial $f \in \mathbf{Q}[x]$, we denote by $\mathbf{Q}(f)$ its splitting field over $\mathbf{Q}$. The same notation is used for an automorphism of $\mathbf{Q}(f)$ and for its natural extension to $\mathbf{Q}(f)^{n}$. Since the following lemma will also be employed in the next section, we do not assume $\Sigma$ to be an ID semigroup.

LemMA II.5. Suppose $\Sigma$ is a commutative semigroup of endomorphisms of $\Omega_{a}^{r}$, containing some $\sigma$ with $f_{\sigma}$ irreducible over $\mathbf{Q}$. Then:

(1) The roots $\lambda_{1, \sigma}, \lambda_{2, \sigma}, \ldots, \lambda_{r . \sigma}$ of $f_{\sigma}$ are mutually distinct.

(2) There exists a basis $v^{(1)}, v^{(2)}, \ldots, v^{(r)}$ of $\mathbf{A}^{r}$ such that

(a) $\Sigma$ is in diagonal form with respect to this basis,

(b) $v^{(i)} \in \mathbf{Q}\left(\lambda_{i, \sigma}\right)^{r}$ for $1 \leqslant i \leqslant r$,

(c) an element $\psi$ of the Galois group of the extension $\mathbf{Q}\left(f_{\mathbf{\sigma}}\right) / \mathbf{Q}$ takes $v^{(i)}$ to $v^{(j)}$ iff it takes $\lambda_{i, \sigma}$ to $\lambda_{j . \sigma}$.

(3) Every $\tau \in \Sigma$ can be uniquely expressed in the form $\tau=\sum_{i=0}^{r-1} a_{i} \boldsymbol{\sigma}^{i}$ with $a_{1} \in \mathbf{Q}$, $0 \leqslant i \leqslant r-1$.

For the proof see [1, Lemma 3.4].

We shall now prove the necessity of the second condition in Theorem II.1. Select some $\sigma \in \Sigma$ with $f_{\sigma}$ irreducible over $\mathbf{Q}$. Let $v^{(1)}, v^{(2)} \ldots v^{(r)}$ be a basis of $\mathbf{A}^{r}$ with the properties stated in the last lemma. For $0 \leqslant j \leqslant h$ denote by $K_{j}$ the splitting field of $f_{\sigma}$ over $\mathbf{Q}_{p} \cdot v^{1 . j}, v^{2.1}, \ldots, v^{r . j}$ is a basis of $K_{j}^{r}$ corresponding to the given basis of $\mathbf{A}^{r}$. The eigenvalues of any $\tau \in \Sigma$, considered as a linear transformation of $K_{j}^{r}$, with respect to this basis will be denoted by $\lambda_{1, j, \tau}, \lambda_{2, j, \tau}, \ldots, \lambda_{r, j, \tau}$.

Proposition II.2. For every $1 \leqslant i \leqslant r$ and $0 \leqslant j \leqslant h$ there exists $a \sigma \in \Sigma$ such that $\left|\lambda_{i, j, o}\right|>1$.

Proof. Suppose that for some $j$ we have, say, $\left|\lambda_{1, j . \sigma}\right| \leqslant 1$ for every $\sigma \in \Sigma$. Assume that $v^{1 . j}, v^{2 . j}, \ldots, v^{k . j}$, for instance, are all the conjugates of $v^{1 . j}$ over $\mathbf{Q}_{p_{j}}$. Then $\lambda_{1, j, \sigma}, \lambda_{2, j, \sigma}, \ldots, \lambda_{k, j, \sigma}$ are all the conjugates of $\lambda_{1, j, \sigma}$ over $\mathbf{Q}_{p_{1}}$ for every $\sigma \in \Sigma$. Since $\mathbf{Q}_{p}$, is complete in the $p_{j}$-adic metric we have

$$
\left|\lambda_{1, j, \sigma}\right|=\left|\lambda_{2, j, \sigma}\right|=\cdots=\left|\lambda_{k, j, o}\right|, \quad \sigma \in \Sigma,
$$

and in particular all these numbers are at most 1.

It is easy to verify that an element of the form $\sum_{l=1}^{r} \alpha_{i} v^{i .1}, \alpha_{i} \in K_{\text {, for }} 1 \leqslant i \leqslant r$, belongs to $\mathbf{Q}_{p_{1}}^{r}$ iff $\alpha_{1}, \alpha_{2}, \ldots, \alpha_{r}$ are conjugate over $\mathbf{Q}_{p_{1}}$ in correspondence with the conjugacy of $v^{1, j}, v^{2, j}, \ldots, v^{r . j}$, i.e. iff $\psi\left(v^{i_{1} . j}\right)=v^{i_{2} . j}$ for some $\psi$ in the Galois group of $K_{j} / \mathbf{Q}_{p}$ implies $\psi\left(\alpha_{i_{1}, j}\right)=\alpha_{i_{2}, j}$. The projection of the set

$$
\mathscr{E}_{R}=\mathbf{Q}_{p_{l}}^{r} \cap\left\{\left.\sum_{i=1}^{h} \alpha_{i} v^{i, j}\left|\alpha_{i} \in K_{j},\right| \boldsymbol{\alpha}_{i}\right|_{j} \leqslant R \forall 1 \leqslant i \leqslant k\right\} \subseteq \mathbf{Q}_{p_{,}}^{r}
$$

on $\Omega_{a}^{r}$ is a $\Sigma$-invariant set in $\Omega_{a}^{r}$ for any $R>0$. This projection is an infinite closed set and for sufficiently small $R>0$ it is properly contained in $\Omega_{a}^{r}$. The contradiction to $\Sigma$ being ID proves the proposition. 
Given a nonsingular matrix $\sigma$ we denote by $G(\sigma)$ the group composed of all integral powers of $\sigma$ and by $S(\sigma)$ the semigroup consisting of all the nonnegative ones. The following lemma forms the first stage towards the proof of the necessity of the third condition in Theorem II.1.

Lemma II.6. Let $\sigma \in \operatorname{End}\left(\Omega_{a}^{r}\right)$. Then

(1) $S(\sigma)$ is not an ID semigroup.

(2) If $\sigma$ is invertible $G(\sigma)$ is not ID.

Proof. We may assume $f_{\sigma}$ to be irreducible over $Q$. An infinite closed proper subset of $\Omega_{a}^{r}$, invariant under the semigroup in question, has to be constructed. We distinguish between two cases:

Case I. $\sigma$ is not an automorphism.

It may be assumed that $\left|\lambda_{i, j, o}\right|>1$ for every $1 \leqslant i \leqslant r, 0 \leqslant j \leqslant h$. Pick a nonzero point $e$ in $\pi^{-1}(\{0\})$, where $\pi$ is the projection of $\prod_{j=0}^{h} \mathbf{Q}_{p}^{r}$ on $\Omega_{a}^{r}$. Decompose $e$ into a sum of eigenvectors of $\sigma$ :

$$
e=\sum_{j=0}^{h} \sum_{i=1}^{r} \alpha_{i, j} v^{i, j} .
$$

It is easy to see that the projection on $\Omega_{a}^{r}$ of the set

$$
\mathcal{O}_{\sigma}^{-}(e)=\left\{\sigma^{-n}(e) \mid n=0,1,2, \ldots\right\} \subseteq \prod_{j=0}^{h} \mathbf{Q}_{p,}^{r}
$$

has the required properties.

Case II. $\sigma$ is an automorphism.

We may assume that $\left|\lambda_{i, j, \sigma}\right| \neq 1$ for $1 \leqslant i \leqslant r, 0 \leqslant j \leqslant h$.

Let $A$ and $B$ denote the sets of pairs $(i, j)$ with $\left|\lambda_{i, j . \sigma}\right|<1$ and with $\left|\lambda_{i . j .0}\right|>1$, respectively. Select $e$ as in the former case and, employing the decomposition (3), take the point

$$
v=e-\sum_{(i, j) \in A} \boldsymbol{\alpha}_{i, j} v^{i, j}=\sum_{(i, j) \in B} \boldsymbol{\alpha}_{i, j} v^{i \cdot j}
$$

Using both representations of $v$ one can show that the projection on $\Omega_{a}^{r}$ of

$$
\mathcal{O}_{\sigma}(v)=\left\{\sigma^{n}(v) \mid n \in \mathbf{Z}\right\} \subseteq \prod_{j=0}^{h} \mathbf{Q}_{p,}^{r}
$$

is infinite and that 0 is its only accumulation point. It follows that the closure of this set possesses the needed properties. This completes the proof.

PROPOSITION II.3. $\Sigma$ contains a pair of rationally independent endomorphisms.

Proof. Choose an endomorphism $\sigma \neq \pm I$ in $\Sigma$ with $f_{\sigma^{n}}$ irreducible over $\mathbf{Q}$ for all $n \in \mathbf{N}$. Denote by $D_{R}(\sigma)$ the set of all matrices with entries in the ring $R$, commuting with $\sigma$ and rationally dependent of it. Lemma II.5 implies that $D_{\mathbf{Q}}(\sigma)$ is a commutative group. We have to show that its subsemigroup $D_{\mathrm{Z}_{[1 / a]}}(\sigma)$ is not ID.

First we claim that $D_{\mathbf{Z}[1 / a]}(\sigma)$ can be represented in the form

$$
D_{\mathbf{Z}[1 / a]}(\sigma)=\bigcup_{i=1}^{s} G(\sigma) \sigma_{i}
$$


if $\sigma$ is an automorphism and in the form

$$
D_{\mathbf{Z}[1 / a]}(\sigma)=\bigcup_{i=1}^{s} S(\sigma) \sigma_{i}
$$

if $\sigma$ is not an automorphism for suitable $\sigma_{1}, \sigma_{2}, \ldots, \sigma_{s}$. Once such a decomposition is given the proposition is proved. In fact, let $E$ be an infinite closed $G(\sigma)$-invariant (or $S(\sigma)$-invariant) proper subset of $\Omega_{a}^{r}$, as constructed in the former lemmas. The conditions imposed upon $\sigma$ guarantee its ergodicity, whence $E$ is nowhere dense (see Chapter I, §2). The set $\bigcup_{i=1}^{s} \sigma_{i}(E)$ is therefore an infinite closed $D_{\mathbf{Z}_{[1 / a]}}(\sigma)$-invariant proper subset of $\Omega_{a}^{r}$, implying that $D_{\mathbf{Z}[1 / a]}(\sigma)$ is not ID.

It thus remains to be shown that $D_{\mathrm{Z}_{[1 / a]}}(\sigma)$ can be represented as in (4) or (5). Let $\tau \in D_{\mathbf{Q}}(\sigma)$. Suppose

$$
\tau^{l}=\sigma^{m} \quad(l, m \in \mathbf{Z}-\{0\})
$$

In particular

$$
\lambda_{1, \tau}^{\prime}=\lambda_{1, \sigma}^{m} .
$$

The solutions $\lambda_{1, \tau} \in K$ of (7) correspond to the solutions $\tau \in D_{\mathbf{Q}}(\sigma)$ of (6). We have to find therefore the solutions of (7). For this a few notions and results from the theory of algebraic numbers are needed (see, for example, [10, Chapter 3]). Let $T$ be the set of equivalent classes of discrete valuations on $K$ for which $\lambda_{1, \sigma}$ is of norm 1 . The set $S$, consisting of all the other equivalence classes, is finite. Denote by $E_{K}$ the finite group of roots of unity belonging to $K$. According to the generalized Dirichlet unit theorem there exist $S$-units $\varepsilon_{1}, \varepsilon_{2}, \ldots, \varepsilon_{u}$ in $K$ such that any $S$-unit $\eta$ in $K$ can be uniquely written in the form

$$
\eta=\xi \cdot \varepsilon_{1}^{k_{1}} \varepsilon_{2}^{k_{2}} \cdots \varepsilon_{u}^{k_{u}} \quad\left(\xi \in E_{K}, k_{1}, k_{2}, \ldots, k_{u} \in \mathbf{Z}\right) .
$$

Since $\lambda_{1 . \sigma}$ is obviously an $S$-unit we have

$$
\lambda_{1, \sigma}=\xi \cdot \varepsilon_{1}^{k_{1}} \varepsilon_{2}^{k_{2}} \cdots \varepsilon_{u}^{k{ }^{\prime}} .
$$

Clearly $\lambda_{1 . \tau}$ forms a solution of (7) iff it is of the form

$$
\lambda_{1, \tau}=\xi^{\prime} \varepsilon_{1}^{t k_{1}} \varepsilon_{2}^{t k_{2}} \ldots \varepsilon_{u}^{t k_{u}}
$$

for some $\xi^{\prime} \in E_{K}$ and $t \in \mathbf{Z} / k$, where $k$ is the g.c.d. of $k_{1}, k_{2}, \ldots, k_{u}$. We obtain therefore $\left[D_{\mathbf{Q}}(\sigma): G(\sigma)\right]<\infty$.

If $\sigma$ is an automorphism, then $D_{\mathrm{Z}[1 / a]}(\sigma)$ is a group and we conclude that $\left[D_{\mathbf{Z}_{[1 / a]}}(\sigma): G(\sigma)\right]<\infty$, which implies that $D_{\mathbf{Z}_{[1 / a]}}(\sigma)$ can be represented as in (4).

If $\sigma$ is not an automorphism, then to any $\tau \in D_{\mathbf{Z}[1 / a]}(\sigma)$ there corresponds in (8) a nonnegative $t$. Selecting from each coset of $D_{\mathrm{Q}}(\sigma)$ modulo $G(\sigma)$ that endomorphism $\tau \in D_{\mathbf{Z}[1 / a]}(\sigma)$ (if there exists any) with minimal $t$, we get a finite set of endomorphisms for which (5) holds. This proves the proposition.

The conditions stated in Theorem II.1 have thus been shown to be necessary for $\Sigma$ to be an ID semigroup. We turn now to the proof of their sufficiency.

3. Sufficiency - sets containing 0 as a nonisolated point. $\Sigma$ is a commutative semigroup of endomorphism of $\Omega_{a}^{r}$ satisfying the conditions of Theorem II.1. $E$ is a closed $\Sigma$-invariant subset of $\Omega_{a}^{r}$ which contains 0 as a nonisolated point. We want to show that $E=\Omega_{a}^{r}$. 
The lifting of $E$ to $\prod_{j=0}^{h} \mathbf{Q}_{p}^{r}$ is also denoted by $E . v^{(1)}, v^{(2)}, \ldots, v^{(r)}$ is a basis of $\mathbf{A}^{r}$ with the properties discussed in Lemma II.5 and $v^{1 . j}, v^{2 . j}, \ldots, v^{r . j}$ is a corresponding basis of $K_{j}^{r}$, where $K_{j}$ is a finite extension of $\mathbf{Q}_{p}, 0 \leqslant j \leqslant h$. The points of $\prod_{j=0}^{h} K_{j}^{r}$ can be represented by $r \times(h+1)$ matrices. For convenience in terminology we shall refer to $\Pi_{j=0}^{h} K_{j}^{r}$ as a vector space, to products of subspaces of the spaces $K_{j}^{r}$ as subspaces of it, and to the vectors $e^{i . j}, 1 \leqslant i \leqslant r, 0 \leqslant j \leqslant h$, of the standard bases of the spaces $K_{j}^{r}$ as its standard basis. The collection of vectors $v^{i, j}, 1 \leqslant i \leqslant r$, $0 \leqslant j \leqslant h$, is the new basis of the space, and with respect to this basis the linear transformations in $\Sigma$ are in diagonal form. A point $v \in \prod_{j=0}^{h} K_{j}^{r}$ belongs to $\prod_{j=0}^{h} \mathbf{Q}_{p}^{r}$, iff in its representation with respect to the new basis $v=\sum_{j=0}^{h} \sum_{i=1}^{r} \alpha_{i, j} v^{i, j}$ the equality $\psi\left(v^{i_{1}, j}\right)=v^{i_{2}, j}$ implies $\psi\left(\alpha_{i_{1}, j}\right)=\alpha_{i_{2}, j}$ for any $0 \leqslant j \leqslant h$ and $\psi$ in the Galois group of $K_{j} / \mathbf{Q}_{p}$. The semigroup obtained from $\Sigma$ by diagonalization is denoted by $\tilde{\Sigma}$ and the matrices corresponding to $\sigma, \tau \in \Sigma$ are denoted by $\tilde{\sigma}, \tilde{\tau} \in \tilde{\Sigma}$. The set of all vectors of coefficients of the points of $E$ represented with respect to the new basis is denoted by $\tilde{E}$. The connection between the representations of vectors and of transformations with respect to the standard basis of $\prod_{j=0}^{h} K_{j}^{r}$ and with respect to the new one is given through the matrix $U$, whose columns are the vectors $v^{(1)}, v^{(2)}, \ldots, v^{(r)}$.

Given a set $A$ of pairs $(i, j)$ we denote by $V_{A}$ the subspace of $\prod_{j=0}^{h} K_{j}^{r}$ spanned by the vectors $e^{i, j}$ with $(i, j) \in A$. Employing the fact that $\tilde{\Sigma}$ contains a matrix. all the diagonal elements of which are distinct, it can be shown that a subspace of $\prod_{j=0}^{h} K_{j}^{r}$ is $\tilde{\Sigma}$-invariant iff it is of the form $V_{A}$ for some $A$. Our assumptions concerning $E$ guarantee that $\tilde{E}$ contains a null sequence of nonzero points. Let $M$ be a minimal set of pairs $(i, j)$ such that $V_{M}$ contains such a sequence $\left(u^{(m)}\right)_{m=1}^{x}$ of points of $\tilde{E}$. Points of $V_{M}$ will be regarded as $|M|$-dimensional vectors.

LEMma II.7. Let $\sigma \in \Sigma$. Then either $\left|\lambda_{i, j, \sigma}\right|>1$ for all $(i, j) \in M$ or $\left|\lambda_{i, j, \sigma}\right| \leqslant 1$ for all $(i, j) \in M$.

Proof. Assume, to the contrary, that for some $\sigma \in \Sigma$ we have $\left|\lambda_{i, j . \sigma}\right|>1$ for $(i, j) \in M_{1}$ and $\left|\lambda_{i, j . \sigma}\right| \leqslant 1$ for $(i, j) \in M_{2}, M_{1}$ and $M_{2}$ being nonvoid complementary subsets of $M$. Put $N(\sigma)=\max _{(i, j) \in M}\left|\lambda_{i, j, \sigma}\right|$. For $a>0$ consider the set

$$
\mathscr{R}_{a}=\left\{\left(z_{i, j}\right)_{(i, j) \in M} \in V_{M}\left|a \leqslant \max _{(i, j) \in M}\right| z_{i, j} \mid \leqslant a N(\sigma)\right\} .
$$

For sufficiently large $m$ there exists a positive integer $j_{m}$ such that $\tilde{\sigma}^{j_{m}}\left(u^{(m)}\right) \in \mathscr{R}_{a}$. Let $w=w(a)$ be a limit point of this sequence. Obviously, $w$ is a nonzero point of $\tilde{E}$ lying in $V_{M_{1}}$. Applying the construction for a sequence $\left(a_{j}\right)_{j=1}^{\infty}$ descending to 0 we obtain a null sequence $\left(w^{(j)}\right)_{j=1}^{\infty}$ of nonzero points of $\tilde{E}$ lying in $V_{M_{1}}$. This contradicts the minimality of $M$ and thereby proves the lemma.

The lemma and the second condition of the theorem imply the existence of some $\sigma \in \Sigma$ for which

$$
\left|\lambda_{i, j, \boldsymbol{o}}\right|>1, \quad(i, j) \in M
$$


Employing a construction similar to the one made in the course of the proof of the last lemma we can show

LEMMA II.8. If $\sigma \in \Sigma$ satisfies (9), then there exists some point $u^{(0)} \in \tilde{E} \cap V_{M}$, all of whose coordinates are nonzero, such that the sequence

$$
u^{(n)}=\tilde{\sigma}^{-n}\left(u^{(0)}\right), \quad n=0,1,2, \ldots,
$$

is a null sequence of points in $\tilde{E} \cap V_{M}$.

LEMMA II.9. For every $\sigma, \tau \in \Sigma$ there exists some $\alpha$ such that

$$
\log \left|\lambda_{i, j, \tau}\right| / \log \left|\lambda_{i, j, \sigma}\right|=\alpha, \quad(i, j) \in M .
$$

Proof. Both $\sigma$ and $\tau$ may be assumed to satisfy (9). Suppose for some nonvoid $M_{1} \subseteq M$ we have

$$
\begin{array}{ll}
\log \left|\lambda_{i, j, \tau}\right| / \log \left|\lambda_{i, j, \sigma}\right|=\alpha, & (i, j) \in M_{1}, \\
\log \left|\lambda_{i, j, \tau}\right| / \log \left|\lambda_{i, j, \sigma}\right|<\alpha, & (i, j) \in M-M_{1} .
\end{array}
$$

Let $\left(u^{(n)}\right)_{n=0}^{\infty}$ be as in the preceding lemma. Define

$$
w^{k . l}=\tilde{\tau}^{k-l}\left(u^{([k \alpha])}\right), \quad k \geqslant l \geqslant 0 .
$$

Denoting by $v_{i, j},(i, j) \in M$, the components of any $v \in V_{M}$ we obtain

$$
w^{k, l}=\sum_{(i, j) \in M} \lambda_{i, j, \tau}^{k-l} \lambda_{l, j, o}^{-[k \alpha]} u_{i, j}^{(0)} e^{i, j}
$$

It follows from (11) that the sequence $\left(w^{k . l}\right)_{k=l}^{\infty}$ of points of $\tilde{E}$ is bounded for any $l$, and has some limit point $w^{(l)}$ lying in $V_{M_{1}}$. Since $\left(w^{(l)}\right)_{l=1}^{\infty}$ is evidently a null sequence of points in $\tilde{E}$, the minimality of $M$ implies $M_{1}=M$, which completes the proof.

In the next lemma we employ the functions exp and log on finite extensions of $\mathbf{Q}_{p}$. The definitions and basic properties of these functions can be found in [10, pp. 206-207]. It will also be convenient to consider exp as the usual isomorphism of $\mathbf{T}$ onto the unit circle in the complex plane, i.e. $\exp (\theta)=e^{2 \pi \imath \theta}$, and $\log$ as its inverse.

Denote $M_{j}=\{i(i, j) \in M\}, 0 \leqslant j \leqslant h . m_{j}$ is the number of elements of $M_{j}$.

Lemma II.10. There exist some $0 \leqslant s \leqslant h$ and elements $\xi_{i} \in K_{s}$ for $i \in M_{s}$, not all of which are 0 , such that for every $t \in \mathbf{Z}_{s}$ the vector

$$
w^{(t)}=u^{(0)}+\sum_{i \in M_{s}}\left(\exp \left(\xi_{t} t\right)-1\right) u_{i, s}^{(0)} e^{i, s}
$$

lies in $\tilde{E}$, where $u^{(0)}$ is as in (10).

Proof. Select two rationally independent endomorphisms $\sigma$ and $\tau$ in $\Sigma$. We may assume that both of them satisfy (9). Let $\alpha$ be as in Lemma II.9. If $\alpha$ is irrational, then $V_{M}$ is a vector space over $\mathbf{R}$ or over $\mathbf{C}$. In this case the proof of the analogous lemma in the toral case [1, Lemma 4.5] is still applicable. Hence we assume $\alpha$ to be rational. Passing to appropriate powers of $\sigma$ and $\tau$ we may assume that $\alpha=1$.

Consider the sequence $\left(w^{(k)}\right)_{k=0}^{\infty}$ obtained from (12) in the special case $l=0$ :

$$
w^{(k)}=\tilde{\tau}^{k}\left(u^{(k)}\right), \quad k \geqslant 0 .
$$


We have

$$
w^{(k)}=\sum_{(i, j) \in M} \lambda_{i, j, \tau}^{k} \lambda_{i, j, \sigma}^{-k} u_{i, j}^{(0)} e^{i, j}=\sum_{(i, j) \in M} \rho_{i, j}^{k} u_{i, j}^{(0)} e^{i, j},
$$

where $\rho_{i, j}$ is a unit in $K_{j}$ for $(i, j) \in M$.

For $j \geqslant 1$ the following notations will be employed. $e_{j}$ is the index of ramification of $K_{j}$ over $\mathrm{Q}_{p_{j}}, R_{j}$ is the ring of integers of $K_{j}, \mathfrak{B}_{j}$ is the prime ideal of $R_{j}$ and $U_{j, m}$ is the multiplicative group $1+\mathfrak{B}_{j}^{m}$. Recall that in the descending sequence of subgroups of the multiplicative group $R_{j}^{*}$ of units in $R_{j}$,

$$
R_{j}^{*} \supseteq U_{j, 1} \supseteq U_{j, 2} \supseteq \cdots,
$$

each group is of finite index in its predecessor [10, p. 203]. Passing to an appropriate power of $\sigma$ and $\tau$ it may be assumed therefore that $\rho_{i, j} \in U_{j\{e, /(p,-1)\}+1}$ for $1 \leqslant j \leqslant h$. It is thus possible to set

$$
\boldsymbol{\theta}_{i, j}=\log \rho_{i, j}, \quad(i, j) \in M .
$$

By (14) we have

$$
w^{(k)}=\sum_{(i, j) \in M} \exp \left(k \theta_{i, j}\right) u_{i, j}^{(0)} e^{i . j}
$$

Consider the set $\left\{\left(k \theta_{i, j}\right)_{(i, j) \in M} \mid k=0,1,2, \ldots\right\}$ of vectors in the group $\mathbf{T}^{m_{0}} \times R_{1}^{m_{1}}$ $\times \cdots \times R_{h}^{m_{h}}$. Its closure is a subgroup, which is infinite since $\sigma$ and $\tau$ are rationally independent. It can be shown that such a subgroup contains a subgroup of the form $\{0\} \times \cdots \times H_{s} \times \cdots \times\{0\}$, where $H_{s}$ is a subgroup of $\mathbf{T}^{m_{0}}($ if $s=0)$ or of $R_{s}^{m_{s}}$ (if $s \geqslant 1$ ), the form of which is $\mathbf{Z}_{p_{s}} \xi$ with $0 \neq \xi \in \mathbf{Z}^{m_{0}}$ or $0 \neq \xi \in \mathbf{Z}_{p_{s}}^{m_{s}}$, respectively. Passing to the limit in (16) we obtain the desired conclusion.

Let $A$ and $B$ be subsets of a metric space $(X, d) . B$ forms an $\varepsilon$-net for $A$ if for every $a \in A$ there exists some $b \in B$ with $d(a, b)<\varepsilon$. A subset $A$ of $V_{M}$ is a line segment if it is of the form

$$
A=a+\{0\} \times \cdots \times \mathbf{Z}_{p_{s}} b \times \cdots \times\{0\},
$$

where $a \in V_{M}$ and $0 \neq b \in K_{s}^{m_{s}}$ for some $0 \leqslant s \leqslant h$. The number $\max _{i \in M_{s}}\left|b_{i}\right|_{s}$ is the length of the segment.

LeMma II.11. For every $\varepsilon>0$ the set $\tilde{E}$ forms an $\varepsilon$-net for arbitrarily long line segments in $V_{M}$.

Proof. Let $\left\{w^{(t)} \mid t \in \mathbf{Z}_{p_{s}}\right\}$ be an "arc" in $\tilde{E}$ as given by the preceding lemma. If $s=0$, then the proof is similar to that of the toral case [1, Lemma 4.6], so we shall deal only with the case $s \geqslant 1$, say $s=1$. For an endomorphism $\sigma \in \Sigma$ satisfying (9) set

$$
w^{m \cdot t}=\tilde{\sigma}^{m}\left(w^{(t)}\right), \quad t \in \mathbf{Z}_{p_{1}}, m=0,1,2, \ldots
$$

By (13) we have

$$
w^{m . t}=\tilde{\sigma}^{m}\left(u^{(0)}\right)+\sum_{i \in M_{1}} \lambda_{i, 1, \sigma}^{m}\left(\exp \left(\xi_{i} t\right)-1\right) u_{i, 1}^{(0)} e^{i .1}
$$


Put

$$
v^{m . t}=\tilde{\sigma}^{m}\left(u^{(0)}\right)+\sum_{i \in M_{1}} \lambda_{i, 1,0}^{m} \xi_{i} t u_{i, 1}^{(0)} e^{i, 1}
$$

for $t \in \mathbf{Z}_{p_{1}}$ and $m=0,1,2, \ldots$ We have

$$
w_{i, 1}^{m, t}-v_{i, 1}^{m, t}=\lambda_{i, 1, \mathrm{o}}^{m}\left(\exp \left(\xi_{i} t\right)-1-\xi_{i} t\right), \quad i \in M_{1} .
$$

To estimate this expression we first show that if $y \in K_{1}$ satisfies

$$
n_{1}(y) \geqslant 3 e_{1} /\left(p_{1}-1\right),
$$

where $n_{1}$ is the exponent corresponding to the ideal $\mathfrak{B}_{1}$, then

$$
|\exp (y)-1-y|_{1} \leqslant\left|y^{2} / 2 !\right|_{1} .
$$

In fact, for such $y$ we have

$$
\begin{aligned}
n_{1}\left(y^{\prime} / l !\right) & =\ln _{1}(y)-n_{1}(l !)=\ln _{1}(y)-e_{1} \sum_{g=1}^{\infty}\left[l / p_{1}^{g}\right] \\
& \geqslant \ln _{1}(y)-e_{1} l /\left(p_{1}-1\right) \geqslant 2 n_{1}(y) \geqslant n_{1}\left(y^{2} / 2 !\right), \quad l \geqslant 3,
\end{aligned}
$$

which, since $\mid \cdot l_{1}$ is nonarchimedean, implies (18).

Obviously, it may be assumed that $n_{1}\left(\xi_{i}\right) \geqslant 3 e_{1} /\left(p_{1}-1\right)$ for $i \in M_{1}$. Let $\|\cdot\|$ be any norm on $V_{\{1\} \times M_{1}}$. Assuming, for example, that $1 \in M_{1}$ and that $\left|\lambda_{1,1,0}\right|$ is maximal among all the elements $\left|\lambda_{i, 1, o}\right|$ with $i \in M_{1}$, we get

$$
\left\|w^{m, t} i_{.} v^{m, t}\right\| \leqslant c\left|\lambda_{1,1, \sigma}\right|^{m}|t|_{1}^{2}, \quad t \in \mathbf{Z}_{p_{1}}, m=0,1,2, \ldots,
$$

for a suitable constant $c$.

Now consider the arcs

$$
\tilde{\mathscr{E}}^{(m)}=\left\{w^{m, t} \mid t \in p_{1}^{s_{m}} \mathbf{Z}_{p_{1}}\right\} \subseteq \tilde{E}, \quad m=0,1,2, \ldots,
$$

and the corresponding line segments

$$
\tilde{\mathcal{I}}^{(m)}=\left\{v^{m, t} \mid t \in p_{1}^{s_{m}} \mathbf{Z}_{p_{1}}\right\}, \quad m=0,1,2, \ldots,
$$

where $\left(s_{m}\right)_{m=1}^{\infty}$ is a sequence of positive integers to be determined later. First, for $\tilde{\mathscr{E}}^{(m)}$ to form an $\varepsilon$-net for $\tilde{\mathscr{I}}^{(m)}$ for sufficiently large $m$ it is sufficient in view of (19) to have

$$
\left|p_{1}\right|_{1}^{2 s_{m}}\left|\lambda_{1,1,0}\right|^{m} \rightarrow 0 \text { as } m \rightarrow \infty .
$$

From (17) it follows that a sufficient condition for the length of $\tilde{\mathcal{I}}^{(m)}$ to tend to infinity with $m$ is that

$$
\left|p_{1}\right|_{1}^{s_{m}}\left|\lambda_{1,1, \sigma}\right|^{m} \rightarrow \infty \quad \text { as } m \rightarrow \infty .
$$

Since $\left(s_{m}\right)_{m=0}^{\infty}$ can evidently be chosen so as to satisfy both (20) and (21) the lemma follows.

Proposition II.4. E contains a translate of some infinite closed $\mathrm{Z}[1 /$ a $]$-submodule of $\Omega_{a}^{r}$.

We review the proof. Let $\tilde{\mathscr{E}}^{(m)}$ and $\tilde{\mathscr{I}}^{(m)}, m \geqslant 0$, be the sets defined in the course of the proof of Lemma II.11, $\mathscr{E}^{(m)}$ and $\mathscr{I}^{(m)}$ the corresponding sets before the 
diagonalization. It is clear that for every $\varepsilon>0, \mathscr{E}^{(m)}$ forms an $\varepsilon$-net for $\mathscr{I}^{(m)}$ for sufficiently large $m$ and that the length of $\mathscr{I}^{(m)}$ tends to infinity with $m$. We claim that $\mathscr{I}^{(m)} \subseteq \Pi_{j=0}^{h} \mathbf{Q}_{p}^{r}$. In fact, we only have to show that if, for instance, $\psi\left(v^{1, j}\right)=v^{2, j}$ for some $\psi$ in the Galois group of $K_{j} / \mathbf{Q}_{p}$, then $\psi\left(v_{1, j}^{m, t}\right)=v_{2, j}^{m, t}$. Since $u^{(0)} \in \tilde{E}$ we have $\psi\left(u_{1, j}^{(0)}\right)=u_{2, j}^{(0)}$. We also have $\psi\left(\lambda_{1, j, \sigma}\right)=\lambda_{2, j, \sigma}$ for every $\sigma \in \Sigma$ and hence $\psi\left(\rho_{1, j}\right)=\rho_{2, j}$, where $\rho_{i, j}$ is determined by (14). This implies $\psi\left(\theta_{1, j}\right)=\theta_{2, j}, \theta_{i, j}$ being defined in (15). It follows that $\psi\left(\xi_{1}\right)=\xi_{2}$ for the elements $\xi_{i}$ in (13). Thus we have $\psi\left(v_{1, j}^{m, t}\right)=v_{2, j}^{m, t}$.

Compactness considerations prove that the projections of the line segments $\mathscr{I}^{(m)}$ on $\Omega_{a}^{r}$ have a subsequence converging in the Hausdorff metric to some translate of an infinite closed $\mathbf{Z}[1 / a]$-submodule of $\Omega_{a}^{r}$. This limit is contained in $E$. This completes the proof.

For the proof of the next lemma see [1, Lemma 4.7].

LEMMA II.12. Let $G$ be a compact abelian group and $\Gamma=\hat{G}$. A sequence $\left(G_{m}\right)_{m=1}^{\infty}$ of closed subgroups of $G$ satisfies $G_{m} \rightarrow G$ as $m \rightarrow \infty$ (in the Hausdorff metric) iff for every $0 \neq \gamma \in \Gamma$ we have $\gamma \notin A\left(\Gamma, G_{m}\right)$ for sufficiently large $m$.

Proposition II.5. Let $\sigma$ be an endomorphism of $\Omega_{a}^{r}$ with $f_{\sigma^{n}}$ irreducible over $\mathbf{Q}$ for every positive integer $n$ and $H$ an infinite closed $\mathrm{Z}[1 / a]$-submodule of $\Omega_{a}^{r}$. Then $\sigma^{m}(H) \rightarrow \Omega_{a}^{r}$ as $m \rightarrow \infty$.

Proof. In view of the preceding lemma we only have to show that if $0 \neq \gamma \in$ $\mathbf{Z}[1 / a]^{r}$, then $\gamma \notin A\left(\mathbf{Z}[1 / a]^{r}, \sigma^{m}(H)\right)$ for sufficiently large $m$. Assume, to the contrary, that $\gamma \in A\left(\mathbf{Z}[1 / a]^{r}, \sigma^{m_{k}}(H)\right)$ for every $k$, where $\left(m_{k}\right)_{k=1}^{\infty}$ is an increasing sequence of positive integers. Then $\gamma \sigma^{m_{k}} \in A\left(\mathbf{Z}[1 / a]^{r}, H\right)$ for all $k$. Hence there exists some $0 \neq \delta \in Z\left[Z[1 / a]^{r}\right.$ such that $\gamma \sigma^{m_{k}} \delta^{T}=0$ for all $k$. Obviously, we may assume that $\gamma, \delta \in \mathbf{Z}^{r}$ and $\sigma \in M_{r}(\mathbf{Z})$. The sequence $\left(\gamma \sigma^{m} \delta^{T}\right)_{m=1}^{\infty}$ of integers satisfies the recursion determined by the polynomial $f_{\sigma}$. Since $f_{\sigma^{n}}$ is irreducible over $\mathbf{Q}$ for every $n \in \mathbf{N}$ no root $\lambda_{i, \sigma}$ and no ratio $\lambda_{i, \sigma} / \lambda_{j, \sigma}$ is a root of unity (unless $r=1$, in which case the theorem is trivial). By [2, p. 302, Problem 9], we have $\gamma \sigma^{m} \delta^{T}=0$ for every $m \in \mathbf{N}$. Hence the $\sigma$-invariant subspace of $Q^{r}$ spanned by $\left\{\sigma^{m} \delta \mid m \in \mathbf{N}\right\}$ is a proper subspace of $\mathbf{Q}^{r}$. The minimal polynomial of the restriction of $\sigma$ to this subspace is a nontrivial divisor of $f_{\sigma}$. This contradicts the irreducibility of $f_{\sigma}$ and thereby proves the proposition.

Combining the conclusions of Propositions II.4 and II.5 we obtain $E=\Omega_{a}^{r}$.

The conditions of Theorem II.1 were thus shown to imply that a closed $\Sigma$ invariant subset of $\Omega_{a}^{r}$ which contains 0 as a nonisolated point is necessarily $\Omega_{a}^{r}$ itself. In the next section the general case is considered.

4. The sufficiency of the conditions. Let $\Sigma$ be a commutative semigroup satisfying the conditions of Theorem II.1. We want to show that $\Sigma$ is an ID semigroup.

Given an abelian group $G$ and a positive integer $l$ we denote by $G[l]$ the subgroup of $G$ consisting of all those elements whose order divides $l$.

LEMMA II.13. $\Omega_{a}^{r}[l]$ is finite for every $l \in \mathbf{N}$. 
Proof. Since $\Omega_{a}^{r}[l] \simeq \mathbf{Z}[1 / a]^{r} / l \mathbf{Z}[1 / a]^{r}$ it is sufficient to show that this quotient group is finite. We shall show, more generally, that if $\Lambda$ is a rank $r$ subgroup of $Q^{r}$, then $\Lambda / l \Lambda$ is a group of order not exceeding $l^{r}$. Let $\gamma_{1}, \gamma_{2}, \ldots, \gamma_{k}$ belong to distinct classes of $\Lambda$ modulo $l \Lambda$. Consider the ascending sequence of subgroups of $\Lambda$ defined by

$$
\Lambda_{n}=\Lambda \cap \frac{1}{n !} \mathbf{Z}^{r}, \quad n=0,1,2, \ldots
$$

$\Lambda_{n}$ is a rank $r$ subgroup of $\mathbf{Z}^{r} / n$ ! and hence it is isomorphic with $\mathbf{Z}^{r}$. Since $\bigcup_{n=0}^{\infty} \Lambda_{n}=\Lambda$ there exists some $n_{0}$ such that $\gamma_{i} \in \Lambda_{n_{0}}$ for $1 \leqslant i \leqslant k$. The elements $\gamma_{1}, \gamma_{2}, \ldots, \gamma_{k}$ are, in particular, distinct modulo $l \Lambda_{n_{0}}$. Now $\mathbf{Z}^{r} / l \mathbf{Z}^{r}$ is a group of order $l^{r}$, so that $k \leqslant l^{r}$. This proves the lemma.

LEMMA II.14. Let $\sigma$ be any epimorphism of $\Omega_{a}^{r}$. $\operatorname{Ker}(\sigma)$ is a finite subgroup of $\Omega_{a}^{r}$.

In fact, for some $l$ we have $l \sigma^{-1} \in M_{r}(\mathrm{Z}[1 / a])$. Obviously $\operatorname{Ker}(\sigma) \subseteq \Omega_{a}^{r}[l]$ for such $l$.

Lemma II.15. Let $\sigma$ be an ergodic endomorphism of $\Omega_{a}^{r}$. A finite $\sigma$-invariant set consists only of torsion elements.

Proof. Any element $x$ in such a set belongs to $\operatorname{Ker}\left(\sigma^{m}-\sigma^{n}\right)$ for some distinct positive integers $m$ and $n$. It is easy to see that the ergodicity criterion of Theorem I.3 implies that $\sigma$ has no roots of unity among its eigenvalues. It follows that $\sigma^{m}-\sigma^{n}$ is an epimorphism. The preceding lemma now implies the desired conclusion.

LEMMA II.16. Let $q$ be a prime not dividing $a$. The subgroup of $\Omega_{a}^{r}$, consisting of all torsion elements whose order is a power of $q$, is dense in $\Omega_{a}^{r}$.

Proof. We claim that, in general, if $G$ is a compact abelian group then the subgroup, consisting of all torsion elements whose order is a power of $q$, is dense iff $\bigcap_{n=0}^{x} q^{n} \hat{G}=\{0\}$. In fact, consider the following ascending sequence of subgroups of $G$ :

$$
G_{n}=G\left[q^{n}\right], \quad n=0,1,2, \ldots
$$

We have

$$
A\left(\hat{G}, G_{n}\right)=q^{n} \hat{G}, \quad n=0,1,2, \ldots
$$

Hence

$$
A\left(\hat{G} \overline{\bigcup_{n=0}^{\infty} G_{n}}\right)=\bigcap_{n=0}^{\infty} q^{n} \hat{G} .
$$

Thus $\cup_{n=0}^{\infty} G_{n}$ is dense in $G$ iff $\bigcap_{n=0}^{\infty} q^{n} \hat{G}=\{0\}$.

In our case, since $q+a$ it is clear that $\bigcap_{n=0}^{\infty} q^{n} \mathbf{Z}[1 / a]^{r}=\{0\}$, whence the lemma follows.

LeMma 11.17. There exists a subsemigroup $\Sigma_{0}$ of $\Sigma$, generated by not more than $\max \{2, r(h+1)\}$ endomorphisms, which also satisfies the conditions of Theorem II.1. 
The proof is simple and bears no difference from the toral case [1, Lemma 5.3].

The following proposition forms the main step of this section. It generalizes Proposition IV.1 of [5] in two respects. First, it relates to semigroups of endomorphisms of $\Omega_{a}^{r}$ instead of $\mathbf{T}^{r}$ and, secondly, it drops the commutativity assumption.

Proposition II.6. Let $\Sigma$ be an arbitrary semigroup of endomorphisms of $\Omega_{a}^{r}$. Suppose that:

(1) There exists some ergodic $\sigma \in \Sigma$.

(2) There exists a prime $q$ not dividing a such that det $\sigma$ is a q-adic unit for every $\sigma \in \Sigma$.

Then $\left(\Omega_{a}^{r}, \Sigma\right)$ is an $\mathscr{F}$-flow.

Proof. For an arbitrary fixed nonnegative integer $k$ denote by $\varphi$ the natural projection of $\mathbf{Z}[1 / a]$ onto $\mathbf{Z}[1 / a] / q^{k} \mathbf{Z}[1 / a]$. The natural extension of $\varphi$ to a projection of $M_{r}(\mathbf{Z}[1 / a])$ onto $M_{r}\left(\mathbf{Z}[1 / a] / q^{k} \mathbf{Z}[1 / a]\right)$ is also denoted by $\varphi$. Let $\Sigma^{(k)}$ be the set of elements of $\Sigma$ carried to $I$ by $\varphi$. Evidently, $\Sigma^{(k)}$ is a subsemigroup of $\Sigma$ and $\Sigma^{(k+1)} \subseteq \Sigma^{(k)}$ for all $k$.

Let $\sigma \in \Sigma$. Since det $\sigma$ is a $q$-adic unit it is invertible modulo $q^{k}$. Hence $\varphi(\sigma)$ is invertible. Denoting by $\Sigma_{1}^{(k)}=\Sigma^{(k)}, \Sigma_{2}^{(k)}, \ldots, \Sigma_{m}^{(k)}$ the inverse images in $\Sigma$ of the elements of $M_{r}\left(\mathbf{Z}[1 / a] / q^{k} \mathbf{Z}[1 / a]\right)$ we see that the second condition in Definition I.4 of $\mathscr{F}$-flows is satisfied.

$\Sigma$ contains some ergodic $\sigma$. For a suitable $l$ we have $\sigma^{\prime} \in \Sigma^{(k)}$. Now $\sigma^{\prime}$ is also ergodic, so that $\left(\Omega_{a}^{r}, \Sigma^{(k)}\right)$ is ergodic, implying the first condition in the definition of $\mathscr{F}$-flows.

It is clear that every torsion element of order $q^{k}$ is a fixed point of $\Sigma^{(k)}$. Lemma II.16 implies therefore that the third condition in the definition of $\mathscr{F}$-flows is also satisfied. This completes the proof.

We now return to study semigroups $\Sigma$ satisfying the conditions of Theorem II.1.

Proposition II.7. Let $M$ be a $\Sigma$-minimal set. $M$ is a finite set of torsion elements.

Proof. By Lemma II.17, $\Sigma$ may be assumed to be finitely generated. Hence $\Sigma$ satisfies the conditions of Proposition II.6. Suppose $M$ is infinite. Then $M-M$ is a closed $\Sigma$-invariant set containing 0 as a nonisolated point. The results of $\S 3$ imply $M-M=\Omega_{a}^{r}$. Since $\left(\Omega_{a}^{r}, \Sigma\right)$ is an $\mathscr{F}$ flow it follows from Theorem I.2 that $M=\Omega_{a}^{r}$. The contradiction proves the proposition.

It is now easy to conclude the proof of Theorem II.1. Let $E$ be an infinite closed $\Sigma$-invariant subset of $\Omega_{a}^{r}$. The set $E^{\prime}$ of accumulation points of $E$ is closed and $\Sigma$-invariant. By the last proposition $E$ contains some torsion element. Hence for a suitable positive integer $l$ the set $l E$ is a closed $\Sigma$-invariant set which contains 0 as a nonisolated point. Hence $l E=\Omega_{a}^{r}$. We infer from this that $E$ has a nonempty interior. $\Sigma$ being ergodic we conclude that $E=\Omega_{a}^{r}$.

The proof of Theorem II.1 is now complete.

REMARK II.2. In [1, §6] the conditions for a commutative semigroup of endomorphisms of $\mathbf{T}^{r}$ to be ID were examined. They were shown to be rather mild and easy to verify in many cases. The same holds for semigroups of endomorphisms of $\Omega_{a}^{r}$. 
We also note the following immediate consequence of Theorem II.1 and Lemma II.17.

THEOREM II.2. Let $\Sigma$ be a commutative ID semigroup of endomorphisms of $\Omega_{a}^{r}$. $\Sigma$ contains a subsemigroup $\Sigma_{0}$, generated by at most $\max \{2, r(h+1)\}$ endomorphisms, which is also ID.

Thus, ID semigroups are not necessarily large. Similarly to the toral case, explicit general examples of ID semigroups generated by just two endomorphisms can easily be given.

CHAPTER III. QUOTIENT GROUPS OF MULTI - SOLENOIDS OF FINITE RANK

1. Generalities and the main theorem. In this chapter the ID property will be studied for commutative semigroups of endomorphisms of quotient groups of $\Omega_{a}^{r}$. First, the groups in this class and their rings of endomorphisms have to be described.

Let $G$ be a quotient group of $\Omega_{a}^{r}$. $\Lambda=\hat{G}$ is a subgroup of $\mathbf{Z}[1 / a]^{r} . \Lambda \cap \mathbf{Z}^{r}$ is isomorphic with $\mathbf{Z}^{s}$ for some $0 \leqslant s \leqslant r$. Let $\psi$ be an isomorphism of $\Lambda \cap \mathbf{Z}^{r}$ onto $Z^{s}$. It is obvious that $\psi$ can be uniquely extended to an isomorphism of $\Lambda$ onto some subgroup of $\mathbf{Z}[1 / a]^{s}$ containing $\mathbf{Z}^{s}$. We may assume therefore that $\mathbf{Z}^{r} \subseteq \Lambda \subseteq$ $\mathbf{Z}[1 / a]^{r}$. Combining this with the endomorphisms introduced in the beginning of the preceding chapter we obtain the monomorphisms

$$
\mathbf{Z}^{r} \stackrel{i_{1}}{\rightarrow} \Lambda \stackrel{i_{2}}{\rightarrow} \mathbf{Z}[1 / a]^{r} \stackrel{i_{3}}{\rightarrow} \mathbf{R}^{r} \times \mathbf{Q}_{a}^{r}
$$

and the dual epimorphisms

$$
\mathbf{T}^{r} \stackrel{\pi_{1}}{\leftarrow} \mathbf{\Omega}_{a}^{r} / H \stackrel{\pi_{2}}{\leftarrow} \mathbf{\Omega}_{a}^{r} \stackrel{\pi_{3}}{\leftarrow} \mathbf{R}^{r} \times \mathbf{Q}_{a}^{r},
$$

where $H$ is a closed subgroup of $\Omega_{a}^{r}$ and $\pi_{j}=\hat{i}_{j}, 1 \leqslant j \leqslant 3$. The mappings $i_{j}$ are discrete embeddings and the mappings $\pi_{j}$ are therefore open. It is easy to verify that

$$
A\left(\mathbf{R}^{r} \times \mathbf{Q}_{a}^{r}, \mathbf{Z}^{r}\right)=\operatorname{Ker}\left(\pi_{3}\right) \oplus\{0\} \times \mathbf{Z}_{a}^{r} .
$$

Hence $\pi_{3}^{-1}(H)=\operatorname{Ker}\left(\pi_{3}\right) \oplus H^{\prime}$, where $H^{\prime}$ is a subgroup of $\mathbf{R}^{r} \times \mathbf{Q}_{a}^{r}$ contained in $\{0\} \times \mathbf{Z}_{a}^{r}$ which is mapped isomorphically onto $H$ by $\pi_{3}$. We shall consequently denote $H^{\prime}$ by $H$.

Recall that $\mathbf{Z}_{a} \simeq \prod_{j=1}^{h} \mathbf{Z}_{p}$, and thus $H$ is a closed subgroup of $\prod_{j=1}^{h} \mathbf{Z}_{p}^{r}$. This can be shown to imply that $H=\prod_{j=1}^{h} H_{j}$, where $H_{j}$ is a closed subgroup of $\mathbf{Z}_{p,}^{r}$ for $1 \leqslant j \leqslant h$. Henceforth, referring to $\Omega_{a}^{r} / H$ we assume that $H$ has the aforementioned properties.

Let $\sigma \in \operatorname{End}\left(\Omega_{a}^{r} / H\right)$. Since $\Lambda \supseteq \mathbf{Z}^{r}$ it is clear that $\hat{\sigma}$ can be uniquely extended to $\mathbf{Z}[1 / a]^{r}$. From Lemma II.1 it follows that $\sigma$ can be uniquely lifted to $\Omega_{a}^{r}$. If $\sigma$ and $\tau$ commute, then so do their liftings to $\Omega_{a}^{r}$ as well. The elements of $\operatorname{End}\left(\Omega_{a}^{r} / H\right)$ are represented by matrices in $M_{r}(\mathbf{Z}[1 / a])$, but not every such matrix gives rise to an endomorphism. The liftings of $\sigma$ to $\Omega_{a}^{r}$ and to $\mathbf{R}^{r} \times \mathbf{Q}_{a}^{r}$ will also be denoted by $\sigma$. Since $H$ is $\sigma$-invariant as a subgroup of $\Omega_{a}^{r}$ and $\{0\} \times \mathbf{Q}_{a}^{r}$ is $\sigma$-invariant in $\mathbf{R}^{r} \times \mathbf{Q}_{a}^{r}$, $H$ is $\sigma$-invariant as a subgroup of $\mathbf{R}^{r} \times \mathbf{Q}_{a}^{r}$ as well. This implies that $H_{j}$ is $\sigma$-invariant 
for $1 \leqslant j \leqslant h$. In fact, we show that $H_{1}$, for example, is $\sigma$-invariant. Let $x \in H_{1}$ and assume that

$$
\sigma(x)=\sum_{j=1}^{h} y_{j}, \quad y_{j} \in H_{j} \forall 1 \leqslant j \leqslant h .
$$

Take a sequence $\left(s_{n}\right)_{n=1}^{\infty}$ of positive integers such that $p_{j}^{s_{n}} \rightarrow 1$ as $n \rightarrow \infty$ in $\mathbf{Z}_{p_{1}}$ for $2 \leqslant j \leqslant h$. Then on the one hand

$$
\sigma\left(\left(p_{2} p_{3} \cdots p_{h}\right)^{s_{n}} x\right) \rightarrow \sigma(x) \text { as } n \rightarrow \infty,
$$

and on the other hand

$$
\sigma\left(\left(p_{2} p_{3} \cdots p_{h}\right)^{s_{n}} x\right)=\sum_{j=1}^{h}\left(p_{2} p_{3} \cdots p_{h}\right)^{s_{n}} y_{j} \rightarrow y_{1} \quad \text { as } n \rightarrow \infty .
$$

Thus $\sigma(x)=y_{1} \in H_{1}$, which proves that $H_{1}$ is $\sigma$-invariant.

Now fix an arbitrary $1 \leqslant j \leqslant h$. Let $V_{j}$ denote the subspace of $\mathbf{Q}_{p}^{r}$ spanned by $H_{j}$. Suppose $V_{j}$ is $s$-dimensional. Obviously, $V_{j}$ is $\sigma$-invariant. Consider the restriction $\sigma_{1}$ of $\sigma$ to $V_{j}$. We claim that all the eigenvalues of $\sigma_{1}$ are of norm not exceeding 1 . Let $K_{j}$ denote the splitting field of $f_{\sigma_{1}}$ over $\mathbf{Q}_{p_{j}}$. Suppose that, contrary to our claim, $v^{(1)} \in K_{j}^{s}$ is an eigenvector of $\sigma_{1}$ with an eigenvalue of norm greater than unity (in the unique extension of the $p_{j}$-adic valuation from $\mathrm{Q}_{p}$, to $\left.K_{j}\right)$. Let $v^{(1)}, v^{(2)}, \ldots, v^{(t)}$ be all the conjugates of $v^{(1)}$ over $\mathbf{Q}_{p}$. All these vectors are eigenvectors of $\sigma_{1}$. Set $x=\sum_{i=1}^{t} v^{(i)}$. It is easy to see that $x \in V_{j}$. Since $H_{j}$ is a $\mathbf{Z}_{p_{j}}$-module we may assume, replacing $x$ by some $p^{k} x$, that $x \in H_{j}$. Now adjoin vectors $v^{(t+1)}, \ldots, v^{(s)}$ in an arbitrary way to $v^{(1)}, \ldots, v^{(t)}$ so as to get a basis of $K_{j}^{s}$. Since $H_{j}$ is compact the set of vectors of coefficients of all the elements of $H_{j}$ with respect to this basis is also compact. But the collection of vectors of coefficients of $\left\{\sigma_{1}^{n}(x) \mid n=0,1,2, \ldots\right\} \subseteq H_{j}$ is already unbounded. The contradiction proves our claim.

The concept of an ID semigroup of endomorphisms of $\Omega_{a}^{r} / H$ is defined as in the case of $\Omega_{a}^{r}$. The following theorem, which generalizes Theorem II.1, forms the main result of the present chapter.

THEOREM III.1. A commutative semigroup $\Sigma$ of endomorphisms of $\Omega_{a}^{r} / H$ is an ID semigroup iff the following conditions are satisfied:

(1) There exists some $\sigma \in \Sigma$ such that $f_{\sigma^{n}}$ is irreducible over $\mathbf{Q}$ for every $n \in \mathbf{N}$.

(2) For every common eigenvector $v$ of $\Sigma$, which is not an eigenvector of the restriction of $\Sigma$ to $\prod_{j=1}^{h} V_{j}$, there exists some $\sigma_{v} \in \Sigma$ whose eigenvalue in the direction of $v$ is of norm greater than unity.

(3) $\Sigma$ contains a pair of rationally independent endomorphisms.

2. The necessity of the conditions. Let $\Sigma$ be a commutative ID semigroup of endomorphisms of $\Omega_{a}^{r} / H$. To prove the necessity of the conditions we have to show that, assuming the conditions to be violated, an infinite closed $\Sigma$-invariant proper subset of $\Omega_{a}^{r} / H$ can be constructed. This set will be the projection of an appropriate subset of $\Omega_{a}^{r}$, as constructed in the course of the proof of Theorem II.1. 
Proposition III.1. There exists some $\sigma \in \Sigma$ with $f_{\sigma^{n}}$ irreducible over $\mathbf{Q}$ for every $n \in \mathbf{N}$.

Proof. In view of the proof of Proposition II.1 it is clearly sufficient to show that the projection of any infinite closed proper $\mathbf{Z}[1 / a]$-submodule of $\Omega_{a}^{r}$ on $\Omega_{a}^{r} / H$ forms an infinite nowhere dense set there.

Let $M$ be such a submodule of $\Omega_{a}^{r}$. Consider the homomorphisms

$$
M \stackrel{i_{1}}{\rightarrow} \Omega_{a}^{r} \stackrel{\pi_{2}}{\rightarrow} \Omega_{a}^{r} / H
$$

and the dual homomorphisms

$$
\mathbf{Z}[1 / a]^{r} / A\left(\mathbf{Z}[1 / a]^{r}, M\right) \stackrel{\pi_{1}}{\leftarrow} \mathbf{Z}[1 / a]^{r} \stackrel{i_{2}}{\leftarrow} \Lambda .
$$

Since $M$ is infinite $A\left(\mathbf{Z}[1 / a]^{r}, M\right)$ is a $\mathbf{Z}[1 / a]$-submodule of infinite index in $\mathbf{Z}[1 / a]^{r}$. Recall that $\mathbf{Z}[1 / a]$ is a Euclidean ring and that every nontrivial submodule is of finite index. The elementary divisor theorem [11, p. 111] implies that $A\left(\mathbf{Z}[1 / a]^{r}, M\right)$ is of rank less than $r$. Since $\Lambda$ is of rank $r, \operatorname{Im}\left(\pi_{1} i_{2}\right)$ is infinite. Hence $\operatorname{Im}\left(\pi_{2} i_{1}\right)$ is also infinite. It remains to show that this set is nowhere dense. To this end note that $A\left(\mathbf{Z}[1 / a]^{r}, M\right)$ is a nontrivial $\mathbf{Z}[1 / a]$-submodule of $\mathbf{Z}[1 / a]^{r}$, whence it is infinite. Rank considerations imply that $\operatorname{Ker}\left(\pi_{1} i_{2}\right)$ is infinite. Consequently the image of $M$ in $\Omega_{a}^{r} / H$ is of infinite index, and so nowhere dense. This proves the proposition.

Employ now the notations introduced before Proposition II.2. Denote by $U_{j}$ the subspace of $K_{j}^{r}$ spanned by $H_{j}$. Our remarks in the beginning of Chapter II, $\S 3$ imply that $U_{j}$ is spanned by some subset of the basis $v^{1, j}, v^{2 . j}, \ldots, v^{r . j}$ of $K_{j}^{r}$. Put

$$
S=\left\{(i, j) \mid 1 \leqslant i \leqslant r, 0 \leqslant j \leqslant h, v^{i, j} \notin U_{j}\right\} .
$$

Note that if $v^{i_{1} \cdot j}$ and $v^{i_{2} . j}$ are conjugate over $\mathbf{Q}_{p}$, and $\left(i_{1}, j\right) \in S$ then $\left(i_{2}, j\right) \in S$ as well.

Proposition III.2. For every $(i, j) \in S$ there exists some $\sigma \in \Sigma$ with $\left|\lambda_{i, j . \sigma}\right|>1$.

Proof. Suppose that for some $j$ we have, say, $(1, j) \in S$ but $\left|\lambda_{1, j . \sigma}\right| \leqslant 1$ for every $\sigma \in \Sigma$. Assume that $v^{1 . j}, v^{2 . j}, \ldots, v^{k . j}$ are the conjugates of $v^{1 . j}$ over $\mathbf{Q}_{p, j}$. Consider the set $\mathscr{E}_{R}$ defined in the proof of Proposition II.2. Under the projection of $\Omega_{a}^{r}$ on $\Omega_{a}^{r} / H$ this set is carried into a closed $\Sigma$-invariant proper subset of $\Omega_{a}^{r} / H$. It is easy to see that $\mathscr{E}_{R}$ is mapped in a 1-1 manner, and therefore its projection is infinite. The contradiction proves the proposition.

PROPOSITION III.3. $\Sigma$ contains a pair of rationally independent endomorphisms.

The proof is carried out employing the constructions made in the proofs of Lemma II.6 and Proposition II.3. We omit the details.

This completes the proof of the necessity.

3. The sufficiency of the conditions. $\Sigma$ is a commutative semigroup which satisfies the conditions of Theorem III.1. We employ the notations introduced before Proposition III.2. 
Proposition III.4. Let $E$ be a closed $\Sigma$-invariant subset of $\Omega_{a}^{r} / H$ containing 0 as a nonisolated point. Then $E=\Omega_{a}^{r} / H$.

Proof. Let $\left(u^{(m)}\right)_{m=1}^{\infty}$ be a nontrivial null sequence of points in $E$. The lifting of $E$ to $\prod_{j=0}^{h} \mathbf{Q}_{p,}^{r}$ contains a null sequence $\left(\bar{u}^{(m)}\right)_{m=1}^{x}$ which forms a lifting of $\left(u^{(m)}\right)_{m=1}^{x}$. Decompose $\bar{u}^{(m)}$ into a sum of eigenvectors in $\prod_{j=0}^{h} K_{j}^{r}$ :

$$
\bar{u}^{(m)}=\sum_{j=0}^{h} \sum_{i=1}^{r} \alpha_{i, j, m} v^{i . j} .
$$

It is easy to see that $\sum_{(i, j) \notin S} \alpha_{i, j, m} v^{i . j} \in \prod_{j=1}^{h} V_{j}$. Since $\bar{u}^{(m)} \rightarrow 0$ as $m \rightarrow \infty$ we have $\alpha_{i, j, m} \rightarrow 0$ as $m \rightarrow \infty$ for every $(i, j)$. Hence for $m$ sufficiently large $\sum_{(i, j) \notin S} \alpha_{i, j, m} v^{i, j}$ $\in H$. The sequence

$$
w^{(m)}=\sum_{(i, j) \in S} \alpha_{i, j, m} v^{i, j}, \quad m=1,2,3, \ldots
$$

therefore forms another lifting of $\left(u^{(m)}\right)_{m=1}^{\infty}$ to $\prod_{j=0}^{h} \mathbf{Q}_{p_{i}}^{r}$. Thus, the lifting of $E$ to $\prod_{j=0}^{h} \mathbf{Q}_{p}^{r}$ contains a nontrivial null sequence of points lying in a $\Sigma$-invariant subspace $V$ of $\prod_{j=0}^{h} \mathbf{Q}_{p}^{r}$, having the following property: For every common eigenvector of the restriction of $\Sigma$ to $V$ there exists some $\sigma \in \Sigma$ with its corresponding eigenvalue of norm greater than unity. Examining the sufficiency part of the proof of Theorem II.1 we observe that this information guarantees that the lifting of $E$ to $\Omega_{a}^{r}$ is $\Omega_{a}^{r}$ itself, and hence $E=\Omega_{a}^{r} / H$.

LEMMA III.1. $\left(\Omega_{a}^{r} / H\right)[l]$ is finite for every $l \in \mathbf{N}$.

The proof of Lemma II.13 is sufficiently general to cover this case also. The following two lemmas are also proved as the parallel ones in the case of $\Omega_{a}^{r}$.

LEMMA III.2. If $\sigma$ is an epimorphism of $\Omega_{a}^{r} / H$, then $\operatorname{Ker}(\sigma)$ is finite.

LEMMA III.3. If $\sigma$ is an ergodic endomorphism of $\Omega_{a}^{r} / H$, then every finite $\sigma$-invariant set consists of torsion elements only.

The following lemma is an immediate consequence of Lemma II.16.

Lemma III.4. Let $q$ be a prime not dividing $a$. Then $\bigcup_{n=0}^{\infty}\left(\Omega_{a}^{r} / H\right)\left[q^{n}\right]$ is dense in $\Omega_{a}^{r} / H$.

Denote by $d\left(H_{j}\right)$ the rank of the $\mathbf{Z}_{p_{l}}$-module $H_{j}$ (or, equivalently, the dimension of $V$, over $\mathbf{Q}_{p}$ ) for $1 \leqslant j \leqslant h$. Set $d(H)=\sum_{j=1}^{h} d\left(H_{j}\right)$. The following generalization of Lemma II.17 holds.

LEMMA III.5. There exists a subsemigroup $\Sigma_{0}$ of $\Sigma$, generated by not more than $\max \{2, r(h+1)-d(H)\}$ endomorphisms, which satisfies also the conditions of Theorem III.1.

Proving that $\Sigma$ is an ID semigroup is now carried out as in the case of $\Omega_{a}^{r}$. It is easy to see that $\left(\Omega_{a}^{r} / H, \Sigma\right)$ may be assumed to be an $\mathscr{F}$-flow. Then we show that a $\Sigma$-minimal set is necessarily a finite set of torsion elements. Finally, it is proved that the only infinite closed set is $\Omega_{a}^{r} / H$. 
REMARK III.1. Unlike what was seen in the former cases, commutative ID semigroups on groups of the form $\Omega_{a}^{r} / H$ are, in some sense, rare. That is, for most subgroups $H$ of $\Omega_{a}^{r}$ the group $\Omega_{a}^{r} / H$ admits no ID semigroups.

EXAMPLE III.1. Consider the group $\Omega_{2}^{2}$. It has a continuum of subgroups obtained as projections of 2-adic segments in $\mathbf{R}^{2} \times \mathbf{Q}_{2}^{2}$, the form of which is $\{(0,0, x, a x) \mid x \in$ $\left.2^{k} \mathbf{Z}_{2}\right\}$ for some $a \in \mathbf{Z}_{2}$ and $k \geqslant 0$. Let $H$ be such a subgroup. If $a$ is not an algebraic number of degree 2 over $Q$ then there exist no ID semigroups on $\Omega_{2}^{2} / H$. If $a=0$, for instance, then the group is $\mathbf{T} \times \Omega_{2} . \mathbf{T} \times\{0\}$ is a fully invariant subgroup, i.e. it is invariant under all the continuous endomorphisms of the group. In fact, since $\bigcup_{n=0}^{\infty} T\left[2^{n}\right]$ is dense in $T$ while $\Omega_{2}[2]=\{0\}$ we have $\operatorname{Hom}\left(\mathbf{T}, \Omega_{2}\right)=\{0\}$. Hence every endomorphism $\sigma$ is represented by a triangular matrix, and so $\mathbf{T} \times\{0\}$ is fully invariant. The situation is similar for other rational numbers $a$. We note that $f_{\sigma}$ is reducible over $\mathbf{Q}$ for every endomorphism $\sigma$. On the other hand, if $a$ is transcendental or algebraic of degree greater than 2 then $\operatorname{End}\left(\Omega_{2}^{2} / H\right)=\{l I \mid l \in \mathbf{Z}\}$. In this case every closed subgroup of $\Omega_{2}^{2} / H$ is fully invariant and $f_{\sigma}$ is reducible for every $\sigma$.

The example shows that the first condition in Theorem III.1 indirectly forms a heavy constraint upon the form of a subgroup $H$ of $\Omega_{a}^{r}$ for which there exist ID semigroups of endomorphisms on $\Omega_{a}^{r} / H$. We note that the case which was seen concerning the group $T \times \Omega_{2}$ can be generalized. To formulate the following theorem we recall a few definitions regarding abelian groups (see, for example, [4, Chapter XII]). Let $\Gamma$ be a torsion-free abelian group. Let $p_{1}, p_{2}, \ldots$ be the sequence of all primes. For $\gamma \in \Gamma$ and prime $p$ denote by $h_{p}(\gamma)$ the largest integer $k$ such that $p^{k} \mid \gamma$; if $p^{k} \mid \gamma$ for every $k$ put $h_{p}(\gamma)=\infty . h_{p}(\gamma)$ is the $p$-height of $\gamma$. The characteristic of $\gamma$ is denoted by $\chi(\gamma)$ and is defined as the sequence of $p$-heights of $\gamma$ :

$$
\chi(\gamma)=\left(h_{p_{1}}(\gamma), h_{p_{2}}(\gamma), \ldots\right) \text {. }
$$

Two characteristics $\left(k_{1}, k_{2}, \ldots\right)$ and $\left(l_{1}, l_{2}, \ldots\right)$ are equivalent if $k_{n}=l_{n}$ for almost all $n$ and $k_{n} \neq l_{n}$ implies that $k_{n}$ and $l_{n}$ are both finite. An equivalence class of characteristics is a type. If $\chi(\gamma)$ is of type $t$ we say that $\gamma$ is of type $t$. The type of $\gamma$ is denoted by $t(\gamma)$. A type $t$ is given by a characteristic in its class. A partial order is defined on the set of all characteristics by setting $\left(k_{1}, k_{2}, \ldots\right) \leqslant\left(l_{1}, l_{2}, \ldots\right)$ if $k_{n} \leqslant l_{n}$ for all $n$. For types we get a partial order by putting $t_{1} \leqslant t_{2}$ if there exist characteristics $\chi_{1} \in t_{1}$ and $\chi_{2} \in t_{2}$ such that $\chi_{1} \leqslant \chi_{2}$. If $\sigma: \Gamma_{1} \rightarrow \Gamma_{2}$ is a homomorphism, then $\chi(\sigma \gamma) \geqslant \chi(\gamma)$ and $t(\sigma \gamma) \geqslant t(\gamma)$ for every $\sigma \in \Gamma$. A group is homogeneous if all its nonzero elements are of the same type.

THEOREM III.2. Let $G$ be a connected compact abelian group. If $\hat{G}$ is not homogeneous, then there exist no ID semigroups of endomorphisms on $G$.

Proof. Suppose $\gamma_{1}, \gamma_{2} \in \hat{G}$ are nonzero elements of different types $t_{1}$ and $t_{2}$, respectively. Assume, say, that $t_{2} \neq t_{1}$. Given any type $t$ we denote $\hat{G}(t)=\{\gamma \in$ $\hat{G} \mid t(\gamma) \geqslant t\}$. $\hat{G}\left(t_{1}\right)$ is a fully invariant subgroup of $\hat{G}$. Our assumptions imply that it is infinite and that it is of infinite index in $\hat{G}$. Hence $A\left(G, \hat{G}\left(t_{1}\right)\right)$ is an infinite closed fully invariant proper subgroup of $G$. This proves the theorem.

We remark that, on the other hand. if commutative ID semigroups on $\Omega_{a}^{r} / H$ exist, then they are not necessarily large. By Theorem III.1 and Lemma III.5 we easily get 
THEOREM III.3. Let $\Sigma$ be a commutative ID semigroup of endomorphisms of $\Omega_{a}^{r} / H$. $\Sigma$ contains a subsemigroup $\Sigma_{0}$, generated by not more than $\max \{2, r(h+1)-d(H)\}$ endomorphisms, which also forms an ID semigroup.

\section{CHAPTER IV. MULTI - SOlENOIDS OF ARBITRARY TYPE}

Generalities and the main theorem. Let $P=\left\{p_{0}, p_{1}, p_{2}, \ldots\right\}$ be a set, where $p_{0}=\infty$ and $p_{j}$ is a prime for $j \geqslant 1$. Denote by $\mathbf{Z}[1 / P]$ the ring obtained from $\mathbf{Z}$ by adjoining all the numbers $1 / p_{j}$ for $j \geqslant 1$. The dual group of the additive group of $\mathrm{Z}[1 / P]$ will be denoted by $\Omega_{P}$. If $P$ is infinite, then $\Omega_{P}$ is called a solenoid of infinite type. For $P=\left\{p_{0}\right\}$ and $P=\left\{p_{0}, p_{1}, \ldots, p_{n}\right\}$ we obtain the circle group and the $a$-adic solenoid (with $a=p_{1} p_{2} \cdots p_{h}$ ), respectively. Our notations in the course of this chapter mostly refer to the infinite case.

We now state a few facts which are analogous to those which were studied for solenoids of finite type. Denote by $\Pi_{j=1}^{\infty} \mathbf{Q}_{p}$, the local direct product of the groups $\mathbf{Q}_{p}$, relative to the compact open subgroups $\mathbf{Z}_{p}, j \geqslant 1$. That is $\prod_{j=1}^{\infty} \mathbf{Q}_{p}$, is the subgroup of $\prod_{j=1}^{\infty} \mathbf{Q}_{p}$, consisting of those elements $\left(x_{j}\right)_{j=1}^{\infty}$ for which $x_{j} \in \mathbf{Z}_{p}$, for almost all $j$. A basis of neighbourhoods of 0 is given by the collection of sets of the form $\prod_{j=1}^{\infty} U_{j}$, where for every $j$ the set $U_{j}$ is a neighbourhood of 0 in $\mathbf{Q}_{p}$, and $U_{j}=\mathbf{Z}_{p_{j}}$ for almost all $j$ (see [7, pp. 56-57] for the definition and the basic properties of local direct products). Put $\prod_{p \in P}^{\prime} \mathbf{Q}_{p}=\mathbf{R} \times \prod_{j=1}^{\infty^{\prime}} \mathbf{Q}_{p}$. The monomorphism $i$ : $\mathbf{Z}[1 / P] \rightarrow \prod_{p \in P}^{\prime} \mathbf{Q}_{p}$ given by $i(s)=(s, s, \ldots), s \in \mathbf{Z}[1 / P]$, is a discrete embedding. Let us examine the dual epimorphism. The group $\Pi_{p \in P}^{\prime} \mathbf{Q}_{p}$ is self-dual $([7,25.34 . b]$ contains this fact as a special case). The action is given by

$$
\left(\left(y_{p}\right)_{p \in P}\right)\left(x_{p}\right)_{p \in P}=\prod_{p \in P} e^{2 \pi i\left\{x_{p}, y_{p}\right\}} .
$$

Hence the dual of $i$

$$
\pi=\hat{i}: \prod_{p \in P}^{\prime} \mathbf{Q}_{p} \rightarrow \Omega_{P}
$$

exhibits $\Omega_{P}$ as a quotient group of $\Pi_{p \in P}^{\prime} \mathbf{Q}_{p}$.

The group $\Omega_{P}$ can also be represented as an inverse limit of solenoids of finite type. If $a / b$ then there exists a natural embedding $\mathrm{Z}[1 / a] \rightarrow \mathbf{Z}[1 / b]$. The system composed of all groups $\mathrm{Z}[1 / a], a$ being a product of primes in $P$, with these embeddings forms a direct system whose limit is $\mathbf{Z}[1 / P]$. Passing to duals we obtain an inverse system whose limit is $\Omega_{P}$.

An explicit representation of $\Omega_{P}$ is given by the set $\prod_{p \in P} \mathbf{Z}_{p}$. If to the elements $x, y \in \Omega_{P}$ there correspond $\left(x_{p}\right)_{p \in P},\left(y_{p}\right)_{p \in P} \in \Pi_{p \in P} \mathbf{Z}_{p}$, then to $z=x+y$ there corresponds $\left(z_{p}\right)_{p \in P}$, where

$$
z_{p}=\left\{\begin{array}{l}
x_{\infty}+y_{\infty}-\left[x_{\infty}+y_{\infty}\right], \quad p=\infty, \\
x_{p}+y_{p}+\left[x_{\infty}+y_{\infty}\right], \quad p \neq \infty .
\end{array}\right.
$$

The projections $\Omega_{b} \rightarrow \Omega_{a}$ for $a \mid b$ and $\Omega_{P} \rightarrow \Omega_{a}$ take on a very simple form in this representation, namely they are defined as projections on the relevant components.

It is easy to see that any endomorphism of $Z[1 / P]^{r}$ can be uniquely extended to $\Pi_{p \in P}^{\prime} \mathbf{Q}_{p}^{r}$. It follows that any endomorphism of $\Omega_{p}^{r}$ can be uniquely lifted to 
$\Gamma_{p \in P}^{\prime} \mathbf{Q}_{p}^{r}$. Endomorphisms of $\Omega_{p}^{r}$ correspond to $r \times r$ matrices over $\mathbf{Z}[1 / P]$. Their liftings act upon $\Pi_{p \in P}^{\prime} \mathbf{Q}_{p}^{r}$ as "linear transformations". The liftings of commuting endomorphisms are also commuting. We note that endomorphisms of $\Omega_{a}^{r}, a$ being a product of primes in $P$, can be uniquely lifted to $\Omega_{P}^{r}$ and to $\Omega_{b}^{r}$ for $a \mid b$.

ID semigroups of endomorphisms of $\Omega_{P}^{r}$ are defined as in the former cases and the characterization of the commutative ones is given by

THEOREM IV.1. A commutative semigroup of endomorphisms of $\Omega_{P}^{r}$ is ID iff it satisfies the three conditions stated in Theorem II.1.

REMARK IV.1. The second condition of the theorem relates, in the case of infinite $P$, to infinitely many eigenvectors; to every $p \in P$ there corresponds a finite extension $K_{p}$ of $\mathbf{Q}_{p}$ such that in $K_{p}^{r}$ there are $r$ common eigenvectors of $\Sigma$. The third condition of the theorem follows in this case from the second one. In fact, this is valid since for every endomorphism $\sigma$ all the eigenvalues $\lambda_{1, \sigma}, \lambda_{2, \sigma}, \ldots, \lambda_{r, \sigma}$ are units in $K_{p}$ for almost all $p$.

2. The proof of the theorem. The necessity of the conditions is proved similarly to the case of finite type multi-solenoids. To obtain the first condition $\mathrm{Z}[1 / P]$-modules are employed instead of the $\mathbf{Z}[1 / a]$-modules of Proposition II.1. For the second we use the projection on $\Omega_{P}^{r}$ of a subset of $\prod_{p \in P}^{\prime} \mathbf{Q}_{p}^{r}$ constructed as in the proof of Proposition II.2. We note that the sets obtained in $\Omega_{P}^{r}$ may be thought of as inverse limits of invariant sets in groups $\Omega_{a}^{r}$. Since it is sufficient to deal with the case of infinite $P$ we obtain by Remark IV.1 the third condition too. Thus we may pass to the proof of the sufficiency of the conditions.

Unlike the preceding cases we do not split the proof into two parts. The main idea is now to make use of projections of $\Sigma$-invariant sets in $\Omega_{P}^{r}$ on quotient groups of $\Omega_{P}^{r}$, which are isomorphic to groups of the form $\Omega_{a}^{r} / H$. From Theorem III.1 we shall conclude that the projection of the given set on any quotient group of that form is the whole of the group. This will imply that this set is $\Omega_{p}^{r}$. A few lemmas are first needed.

LEMMA IV.1. $\Omega_{P}^{r}[l]$ is finite for every $l \in \mathbf{N}$.

The proof of Lemma II.13 covers our case also.

LeMmA IV.2. Let $a$ and $b$ be positive integers with $a \mid b$ and let $\pi: \Omega_{b} \rightarrow \Omega_{a}$ denote the natural projection. If $x \in \Omega_{b}^{r}$ is a torsion element then $\pi(x) \in \Omega_{a}^{r}$ is a torsion element whose order is the same as that of $x$.

Proof. It is sufficient to deal with the case $r=1$. We have to show that if $x \in \Omega_{b}^{r}$ is a torsion element and $\pi(x)=0$ then $x=0$. We may assume that $x \in \Omega_{b}[q]$ for a certain prime $q$. To show that the restriction of $\pi$ to $\Omega_{h}[q]$,

$$
\pi^{\prime}: \Omega_{b}[q] \rightarrow \Omega_{a}[q],
$$

forms a monomorphism, it is sufficient to prove that its dual

$$
\hat{\pi}^{\prime}: \mathbf{Z}[1 / a] / q \mathbf{Z}[1 / a] \rightarrow \mathbf{Z}[1 / b] / q \mathbf{Z}[1 / b],
$$


which is naturally induced by the embedding of $\mathbf{Z}[1 / a]$ into $\mathbf{Z}[1 / b]$, is surjective. Now if $q \mid b$ then the group on the right-hand side is trivial, while if $q+b$, then $\hat{\pi}^{\prime}$ is obviously 1-1 and since both groups are of the same order $q$ it is also onto. This proves the lemma.

Now let $\Sigma$ be a commutative semigroup of endomorphisms of $\Omega_{P}^{r}$ satisfying the conditions of the theorem. $E$ is an infinite closed $\Sigma$-invariant set. $\pi_{a}$ denotes the projection of $\Omega_{P}^{r}$ on $\Omega_{a}^{r}$, where $a$ is a product of primes in $P$.

Proposition IV.1. There exists an a such that $\pi_{a}(E)$ is infinite.

Proof. By the characterization of ergodicity in Theorem I.3 we easily see that $\Sigma$ contains some ergodic endomorphism $\sigma$. Let $a$ be the product of finitely many primes in $P$, including all the primes dividing the denominator of some entry in $\sigma$. Obviously, $\sigma$ induces an ergodic endomorphism on $\Omega_{a}^{r}\left(\left(\Omega_{a}^{r}, \sigma\right)\right.$ is a factor of $\left.\left(\Omega_{P}^{r}, \sigma\right)\right)$. The set $\pi_{a}(E)$ is closed and $\sigma$-invariant. Assume it is finite. Then by Lemma II.14 we have $\pi_{a}(E) \subseteq \Omega_{a}^{r}[l]$ for an appropriate positive integer $l$. Lemma IV.2 implies that $\pi_{b}(E) \subseteq \Omega_{b}^{r}[l]$ for every $b$ which is divisible by $a$. From the representation of $\Omega_{P}^{r}$ as an inverse limit of multi-solenoids of finite type we conclude that $E \subseteq \Omega_{p}^{r}[l]$. Lemma IV.1 proves the finiteness of $E$. The contradiction completes the proof.

Proposition IV.2. For every product a of primes in $P, \pi_{a}(E)=\Omega_{a}^{r}$.

Proof. In view of the preceding proposition we may assume that $\pi_{a}(E)$ is infinite. Suppose, say, that $a=p_{1} p_{2} \cdots p_{h}$. Choose a finite set of endomorphisms $\sigma_{1}, \sigma_{2}, \ldots, \sigma_{s} \in \Sigma$ such that $f_{\sigma_{1}^{n}}$ is irreducible for all $n, \sigma_{2}$ is rationally independent of $\sigma_{1}$ and for every $1 \leqslant i \leqslant r$ and $0 \leqslant j \leqslant h$ there exists some $1 \leqslant t \leqslant s$ with $\left|\lambda_{i, j . \sigma_{t}}\right|>1$. Let $\Sigma_{0}$ be the subsemigroup of $\Sigma$ generated by $\sigma_{1}, \sigma_{2}, \ldots, \sigma_{s}$. Let $b$ be the product of finitely many primes in $P$, including all those primes dividing the denominator of some entry of one of the matrices $\sigma_{1}, \sigma_{2}, \ldots, \sigma_{s}$, say $b=p_{1} p_{2} \cdots p_{h} \cdots p_{k}$.

For every $h+1 \leqslant j \leqslant k$ denote by $H_{j}$ the closed subgroup of $\mathbf{Z}_{p}^{r}$, consisting of those elements lying in the subspace of $K_{j}^{r}$ spanned by the eigenvectors $v^{1, j}, v^{2, j}, \ldots, v^{r, j}$ with $\left|\lambda_{i, j, \sigma}\right| \leqslant 1$ for every $\sigma \in \Sigma_{0}$. The group $H_{j}$ is not necessarily $\Sigma$-invariant, but from compactness considerations we easily infer that $\Sigma_{0} H_{j} \subseteq p_{j}^{-l,} \mathbf{Z}_{p}^{r}$, for a suitable $l_{j}$ (see also the remarks preceding Theorem III.1). Set $H_{j}^{\prime}=p_{j}^{\prime} \bar{\Sigma}_{0} H_{j}$. Evidently, $H_{j}^{\prime}$ is a closed $\Sigma_{0}$-invariant subgroup of $\mathbf{Z}_{p}^{r}$. Hence $\Sigma_{0}$ may be considered as a commutative semigroup of endomorphisms of $\Omega_{h}^{r} / \Pi_{j=h+1}^{k} H_{j}^{\prime}$. One can show that our construction implies that, as such, $\Sigma_{0}$ satisfies the conditions of Theorem III.1, and hence $\left(\Omega_{b}^{r} / \Pi_{j=h+1}^{k} H_{j}^{\prime}, \Sigma_{0}\right)$ is ID. Let $\pi_{b}^{\prime}$ denote the projection of $\Omega_{P}^{r}$ on $\Omega_{b}^{r} / \Pi_{j=h+1}^{k} H_{j}^{\prime}$. The set $\pi_{b}^{\prime}(E)$ is a closed $\Sigma_{0}$-invariant set. Since $\pi_{a}(E)$ is infinite, $\pi_{b}^{\prime}(E)$ is infinite as well. Hence $\pi_{b}^{\prime}(E)=\Omega_{b}^{r} / \Pi_{j=h+1}^{k} H_{j}^{\prime}$, and therefore $\pi_{a}(E)=\Omega_{a}^{r}$. This proves the proposition.

From Proposition IV.2 and the representation of $\Omega_{P}^{r}$ as an inverse limit of groups of the form $\Omega_{a}^{r}$ we immediately obtain $E=\Omega_{P}^{r}$. The proof of Theorem IV.1 is thus concluded.

REMARK IV.2. As in the case of multi-solenoids of finite type the conditions for a commutative semigroup of endomorphisms of a multi-solenoid of infinite type to be 
ID are rather mild and easy to verify. We can also construct explicitly ID semigroups. On the other hand, it is obvious that in the infinite type case no finitely generated semigroup (commutative or not) is ID.

\section{ChAPTER V. FinITE - DIMENSIONAL CONNECTED COMPACT ABELIAN GROUPS}

1. Generalities and the main theorem. This chapter deals with the characterization of commutative ID semigroups of endomorphisms of finite-dimensional connected compact abelian groups. This class of groups contains as special cases the classes studied in the former chapters.

Let $G$ be such a group. $\Gamma=\hat{G}$ is a torsion-free discrete abelian group of finite rank (see, for example, [7, Chapters 23-24]). We may assume therefore that $\mathbf{Z}^{r} \subseteq \Gamma \subseteq \mathbf{Q}^{r}$ for some $r$. To make the notations compatible with those of the preceding chapter we assume that $\Gamma \subseteq \mathbf{Z}[1 / P]^{r}$ for a certain $P$ (which may be considered to include all the primes). Hence $G$ is of the form $\Omega_{P}^{r} / H$.

We have to connect our group with $\Omega_{P}^{r}$ and with quotient groups of multi-solenoids of finite type. Let $a$ be a product of primes in $P$. Consider the commutative diagram of monomorphisms

$$
\begin{array}{ccccccc}
\mathbf{Z}^{r} & \rightarrow & \Gamma \cap \mathbf{Z}[1 / a]^{r} & \rightarrow & \mathbf{Z}[1 / a]^{r} & \rightarrow & \prod_{j=0}^{h} \mathbf{Q}_{p,}^{r} \\
\downarrow & & \downarrow & & \downarrow & & \downarrow \\
\mathbf{Z}^{r} & \rightarrow & \Gamma & \rightarrow & \mathbf{Z}[1 / P]^{r} & \rightarrow & \prod_{p \in P}^{\prime} \mathbf{Q}_{p}^{r}
\end{array}
$$

and the corresponding dual diagram of epimorphisms:

$$
\begin{aligned}
& \mathbf{T}^{r} \leftarrow \Omega_{a}^{r} / \prod_{j=1}^{h} H_{j} \leftarrow \Omega_{a}^{r} \leftarrow \prod_{j=0}^{h} \mathbf{Q}_{p,}^{r} \\
& \uparrow \quad \uparrow \quad \uparrow \quad \uparrow \\
& \mathbf{T}^{r} \leftarrow \Omega_{P}^{r} / H \leftarrow \Omega_{P}^{r} \leftarrow \prod_{p \in P}^{\prime} \mathbf{Q}_{p}^{r}
\end{aligned}
$$

The inverse image of $H$ in $\prod_{p \in P}^{\prime} \mathbf{Q}_{p}^{r}$ contains a subgroup, contained in $\prod_{p \in P-\{\infty\}} \mathbf{Z}_{p}^{r}$, which is carried isomorphically to $H$. This group, which we denote by $H$ also, decomposes in the form $H=\prod_{j=1}^{\infty} H_{j}$, where $H_{j}$ is a closed subgroup of $\mathbf{Z}_{p}^{r}$, for $j \geqslant 1$. If $a$ and $b$ are products of primes in $P$ with $a \mid b$, then we have a natural embedding $\Gamma \cap \mathbf{Z}[1 / a]^{r} \rightarrow \Gamma \cap \mathbf{Z}[1 / b]^{r}$ and a corresponding dual projection $\Omega_{b}^{r} / \prod_{p, \mid h} H_{j} \rightarrow \Omega_{a}^{r} / \prod_{p, \mid a} H_{j}$. Passing over all the products of primes in $P$ we obtain an inverse system whose limit is $\Omega_{P}^{r} / H$.

Let $\sigma \in \operatorname{End}\left(\Omega_{P}^{r} / H\right)$. As in the case of finite $P$ it is easily verified that $\sigma$ admits a unique lifting to $\Omega_{P}^{r}$. The groups $H_{j}, j \geqslant 1$, are invariant under the lifting of $\sigma$ to $\Pi_{p \in P}^{\prime} \mathbf{Q}_{p}^{r}$. The liftings of commuting endomorphisms are commuting as well.

Denote by $V_{j}$ the subspace of $\mathbf{Q}_{p}^{r}$ spanned by $H_{j}$ for $j \geqslant 1$ and $V_{0}=\{0\}$. If $\sigma \in \operatorname{End}\left(\Omega_{p}^{r} / H\right)$, then the invariance of $H_{j}$ under $\sigma$ implies that the eigenvalues of the restriction of $\sigma$ to $V_{j}$ are of norms not exceeding unity. Put

$$
\underline{H}_{j}=V_{j} \cap \mathbf{Z}_{p}^{r}, \quad j \geqslant 1 \text {. }
$$

It is easy to see that $H$, is a subgroup of finite index in $\underline{H}_{j}$. 
The following theorem, which includes Theorems II.1, III.1 and IV.1 as special cases, forms the main result of the whole paper.

THEOREM V.1. A commutative semigroup $\Sigma$ of endomorphisms of $\Omega_{P}^{r} / H$ is ID iff the following conditions hold:

(1) $\underline{H}_{j}=H_{j}$ for almost every $j$.

(2) There exists a $\sigma \in \Sigma$ such that $f_{\sigma^{n}}$ is irreducible over $\mathbf{Q}$ for every $n \in \mathbf{N}$.

(3) For every $j \geqslant 0$ and every common eigenvector $v$ of the semigroup $\Sigma$ of linear transformations of $\mathbf{Q}_{p}^{r}$, which is not an eigenvector of the resiriction of $\Sigma$ to $V_{j}$, there exists an endomorphism $\sigma_{r} \in \Sigma$ whose eigenvalue in the direction of $v$ is of norm greater than unity.

(4) $\Sigma$ contains a pair of rationally independent endomorphisms.

REMARK V.1. The first condition of the theorem relates only to the structure of the group in question. If it is not satisfied then there exist no commutative ID semigroups of endomorphisms on the group. Recall that in the case of $\Omega_{a}^{r} / H$ there were also restrictions on the structure of $H$ required to guarantee the existence of ID semigroups. But whereas in that case the restrictions were implicit in the condition regarding the existence of some $\sigma \in \Sigma$ with $f_{\sigma}$ irreducible, in our case the structure of $\Sigma$ alone cannot ensure that the group admits any ID semigroups (see also Example V.1).

2. The proof of the theorem. We first prove the necessity of the conditions. To obtain conditions (2) and (3) we employ projections of properly chosen invariant sets in $\Omega_{p}^{r}$. The method is the same as the one used in the case of $\Omega_{a}^{r} / H$.

Now we have a basis $v^{(1)}, v^{(2)}, \ldots, v^{(r)}$ of $\mathbf{A}^{r}$ with the properties considered in Lemma II.5. $U$ is the matrix formed by these column vectors. The entries of $U$ and of $U^{-1}$ belong to a certain number field $K$. Let $P_{1}$ denote the set of all primes $p \in P$ for which all the elements of $U$ and of $U^{-1}$ are of norm not exceeding unity for every extension of the $p$-adic valuation from $\mathbf{Q}$ to $K$. The set $P-P_{1}$ is finite. If $p_{j} \in P_{1}$, then a vector $v$ in $\mathbf{Q}_{p}^{r}$, belongs to $\mathbf{Z}_{p}^{r}$ iff in the representation $v=\sum_{i=1}^{r} \alpha_{i, j} v^{i . j}$ we have $\left|\alpha_{i, j}\right| \leqslant 1$ for every $1 \leqslant i \leqslant r$. Hence if $p_{j} \in P_{1}$ then $Z_{p_{1}}$ is $\Sigma$-invariant, and therefore $\underline{H}_{j}$ is also $\Sigma$-invariant. Put $H_{j}^{\prime}=\underline{H}_{j}$ if $p_{j} \in P_{1}$ and $H_{j}^{\prime}=H_{j}$ if $p_{j} \notin P_{1}$. Then $\Pi_{j=1}^{\infty} H_{j}^{\prime}$ is a $\sum$-invariant compact subgroup of $\prod_{p \in P}^{\prime} \mathbf{Q}_{p}^{r}$ containing $\prod_{j=1}^{\infty} H_{j}$. The projection of $\Pi_{j=1}^{\infty} H_{j}^{\prime}$ on $\Omega_{P}^{r} / H$ is a closed $\Sigma$-invariant proper subgroup of $\Omega_{P}^{r} / H$. If for infinitely many indices $j$ the subgroup $H_{j}$ is properly contained in $\underline{H}_{j}$, then this projection is infinite. It follows that condition (1) of Theorem V.1 is necessary for the existence of commutative ID semigroups on $\Omega_{p}^{r} / H$.

We turn to prove the necessity of condition (4). If for infinitely many numbers $j$ the rank of the $\mathbf{Z}_{p}$-module $H_{j}$ is strictly less than $r$, then obviously the third condition implies the fourth. Assume therefore that $H_{j}$ is of rank $r$ for almost every $j$. Then the first condition implies that $H_{j}=\mathbf{Z}_{p}^{r}$, for almost all $j$, so that our group is actually a quotient group of a multi-solenoid of finite type, where the theorem was already proved.

Now the sufficiency of the conditions will be proved. Two lemmas are needed first. 
LEMMA V.1. $\left(\Omega_{p}^{r} / H\right)[l]$ is finite for every $l \in \mathbf{N}$.

The proof of Lemma II.13 applies to this case also.

Given products $a$ and $b$ of primes in $P$ with $a \mid b$ we denote by $\pi_{b, a}$ the projection of $\Omega_{h}^{r}$ on $\Omega_{a}^{r}$ as well as the projection of $\Omega_{h}^{r} / \prod_{p, \mid h} H_{j}$ on $\Omega_{a}^{r} / \prod_{p, \mid a} H_{j}$.

LemMa V.2. Let $a$ and $b$ be as before. Suppose that $H_{j}=\underline{H}_{j}$ for every $j$ for which $p_{j} \mid(b / a)$. Let $x$ be a torsion element of $\Omega_{b}^{r} / \prod_{p, \mid b} H_{j}$. Then $\pi_{b, a}(x)$ is a torsion element of $\Omega_{a}^{r} / \Pi_{p, \mid a} H_{j}$ of the same order as $x$.

Proof. Assume that, say, $a=p_{1} p_{2} \cdots p_{h}$ and $b=p_{1} p_{2} \cdots p_{h} \cdots p_{k}$. It is sufficient to show that $x \in\left(\Omega_{h}^{r} / \prod_{j=1}^{k} H_{j}\right)[l]$ and $\pi_{b . a}(x)=0$ imply $x=0$. Lifting to $\Omega_{h}^{r}$ we see that we have to prove that if $x \in \Omega_{b}^{r}, l x \in \Pi_{j=1}^{k} H_{j}$ and $\pi_{h, a}(x) \in \Pi_{j=1}^{h} H_{j}$, then $x \in \Pi_{j=1}^{k} H_{j}$. Employ the explicit representations of $\Omega_{a}$ and $\Omega_{h}$ as products of suitable $\mathbf{Z}_{p_{j}}$ 's. Our assumptions imply that $x=\left(x_{1}, x_{2}, \ldots, x_{h}, \ldots, x_{k}\right)$ with $x_{j} \in H_{j}$ for $1 \leqslant j \leqslant h$ and $x_{j} \in \mathbf{Z}_{p,}^{r}$ for $h+1 \leqslant j \leqslant k$. Since $x$, belongs to the subspace of $\mathbf{Q}_{p_{1}}^{r}$ spanned by $l x_{j}$ we get $x_{j} \in H_{j}$ for $h+1 \leqslant j \leqslant k$. But $\underline{H}_{j}=H_{j}$ for $h+1 \leqslant j \leqslant k$. We obtain $x_{j} \in H_{j}$ for $1 \leqslant j \leqslant k$. This completes the proof.

Let $\Sigma$ be a commutative semigroup of endomorphisms of $\Omega_{P}^{r} / H$ such that the conditions of the theorem are satisfied. $E$ is an infinite closed $\Sigma$-invariant set. $\pi_{a}$ denotes the projection of $\Omega_{p}^{r} / H$ on $\Omega_{a}^{r} / \prod_{p, \mid u} H_{j}$.

Proposition V.1. There exists some a such that $\pi_{a}(E)$ is infinite.

Proof. Select an ergodic $\sigma \in \Sigma$. Let $a$ be the product of finitely many primes in $P$, including all those primes dividing the denominator of some entry of $\sigma$ and all those primes $p_{j}$ such that $\underline{H}_{j} \neq H_{j}$. Assume that, say, $a=p_{1} p_{2} \cdots p_{h}$. $\sigma$ induces an ergodic endomorphism on $\Omega_{a}^{r} / \prod_{j=1}^{h} H_{j} \cdot \pi_{a}(E)$ is a closed $\sum$-invariant set. If it is finite then $\pi_{a}(E) \subseteq\left(\Omega_{a}^{r} / \Pi_{j=1}^{h} H_{j}\right)[l]$ for some $l$. By Lemma V.2 we obtain $\pi_{b}(E) \subseteq$ $\left(\Omega_{b}^{r} / \Pi_{j=1}^{k} H_{j}\right)[l]$ for every $b$ of the form $b=p_{1} p_{2} \cdots p_{h} \cdots p_{k}$. The representation of $\Omega_{P}^{r} / H$ as an inverse limit of groups of the form $\Omega_{b}^{r} / \Pi_{j=1}^{k} H_{j}$ implies then that $E \subseteq\left(\Omega_{p}^{r} / H\right)[l]$. This contradicts the infinity of $E$ and thereby proves the proposition.

The end of the proof is analogous to that of the case of $\Omega_{P}^{r}$. First it is shown, similarly to Proposition IV.2, that $\pi_{a}(E)=\Omega_{a}^{r} / \Pi_{p, \mid a} H_{j}$ for every $a$. From the representation of $\Omega_{P}^{r} / H$ as an inverse limit of quotient groups of multi-solenoids of finite type we infer that $E=\Omega_{p}^{r} / H$. This completes the proof of Theorem V.1.

REMARK V.2. In the proof we saw that if $P$ is infinite then $\Omega_{P}^{r} / H$ usually admits no finitely generated commutative ID semigroups. In fact, if such semigroups do exist then the group is actually isomorphic to a quotient group of a multi-solenoid of finite type.

We now bring without proof a condition on the dual group $\Gamma$ which is equivalent to the condition $\underline{H}_{j}=H_{j}$. Consider the group

$$
\Gamma_{j}=\Gamma \cap \mathbf{Z}\left[1 / p_{j}\right]^{r} / \mathbf{Z}^{r}, \quad j \geqslant 1 \text {. }
$$

Then $\underline{H}_{j}=H_{\text {, iff }} \Gamma_{j}$ is a divisible group.

EXAMPLE V.1. Let $\Gamma$ be the subgroup of $\mathbf{Q}$ consisting of all rationals with square-free denominator. Obviously $\operatorname{End}(\Gamma) \simeq \mathbf{Z}$. Every subgroup of $\Gamma$ is fully 
invariant. The dual group $G=\hat{\Gamma}$ can be represented by the set $[0,1) \times \prod_{p \in P} C_{p}$, where $P$ is the set of all primes and $C_{p}$ is the cyclic group of order $p$ (addition is defined by transferring the remainder in the first component to all others). The set of all closed subgroups of $G$ has the power of the continuum and each subgroup is fully invariant. More generally, out of all the subgroups of $\mathbf{Q}$, only for those which are isomorphic with $\mathrm{Z}[1 / P]$ for some $P$ do there exist ID semigroups on the dual group. This is a special case of the following

THEOREM V.2. Let $G$ be a connected compact abelian group and let $\Gamma=\hat{G} . A$ necessary condition for the existence of ID semigroups of endomorphisms on $G$ is that $\Gamma$ be a homogeneous group of idempotent type.

Recall that a type $t$ is idempotent if there exists a characteristic $\chi=\left(k_{1}, k_{2}, \ldots\right) \in t$ with $k_{n}=0$ or $k_{n}=\infty$ for all $n$ (see [4, Chapter XII]).

Proof. $\Gamma$ is necessarily homogeneous by Theorem III.2. If $\Gamma$ is not of an idempotent type, then it contains fully invariant infinite subgroups of infinite index. In fact, let $0 \neq \gamma \in \Gamma$. Set

$$
\Lambda=\{\delta \in \Gamma \mid \chi(\delta) \geqslant \chi(\gamma)\} .
$$

$\Lambda$ is an infinite subgroup of $\Gamma$. Since $\Gamma$ is not of an idempotent type, there exist infinitely many primes $p$ such that $p \mid \gamma$ but $p^{k}+\gamma$ for sufficiently large $k$. The collection of all elements $\gamma / p$ for these primes $p$ forms an infinite set of elements which are mutually incongruent modulo $\Lambda$. Hence $\Lambda$ is of infinite index in $\Gamma$. Evidently $\Lambda$ is fully invariant. It follows that $A(G, \Lambda)$ is an infinite closed fully invariant proper subgroup of $G$. This completes the proof.

\section{REFERENCES}

1. D. Berend, Multi-int'ariant sets on tori, Trans. Amer. Math. Soc. 280 (1983), 509-532.

2. Z. I. Borevich and I. R. Shafarevich, Number theon. Academic Press, New York and London, 1966.

3. J. R. Brown, Ergodic theon and topological dynamics, Academic Press. New York and London, 1976.

4. L. Fuchs, Infinite abelian groups, Vol. II, Academic Press, New York and London, 1973.

5. H. Furstenberg, Disjointness in ergodic theon, minimal sets, and a problem in diophantine approximation, Math. Systems Theory 1 (1967), 1-49.

6. Intersections of Cantor sets and transtersality of semigroups, Problems in Analysis ( R. C. Gunning, general ed.), Princeton Univ. Press, Princeton. N. J., 1970, pp. 41-59.

7. E. Hewitt and K. A. Ross, Abstract harmonic analysis. Vol. I, Springer-Verlag. Berlin, 1963.

8. N. Jacobson, Lectures in abstract algebra. Vol. II, Van Nostrand, Princeton, N. J., 1953. 1973.

9. K. Mahler, Introduction to p-adic numbers and their functions, Cambridge Univ. Press. Cambridge,

10. W. Narkiewicz. Elementan and analytic theon of algebraic numbers, Polish Scientific Publishers, Warsaw, 1974.

11. B. L. van der Waerden, Modern algebra. Vol. II, Ungar, New York, 1950.

Department of Mathematics, University of California, Los Angeles, California 90024 